\title{
Radiation Damage and Single Event Effect Results for Candidate Spacecraft Electronics
}

\author{
Martha V. O'Bryan', Kenneth A. LaBel ${ }^{2}$, Robert A. Reed², James W. Howard Jr. ', Ray L. Ladbury ${ }^{4}$, \\ Janet L. Barth ${ }^{2}$, Scott D. Kniffin ${ }^{4}$, Christina M. Seidleck', Paul W. Marshall ${ }^{5}$, Cheryl J. Marshall', \\ Hak S. Kim³ ${ }^{3}$, Donald K. Hawkins ${ }^{2}$, Anthony B. Sanders', Martin A. Carts ${ }^{1}$, James D. Forney, \\ David R. Roth ${ }^{6}$, James D. Kinnison ${ }^{6}$, Elbert Nhan", and Kusum Sahu ${ }^{7}$ \\ 1. Raytheon Information Technology \& Scientific Services, Lanham, MD 20706-4392 \\ 2. NASA/GSFC, Code 562, Greenbelt, MD 20771 \\ 3. Jackson \& Tull Chartered Engineers, Washington. D. C. 20018 \\ 4. Orbital Sciences Corporation, McLean, VA \\ 5. Consultant \\ 6. Applied Physics Laboratory, Laurel, Maryland 20723-6099 \\ 7. QSS, Laurel, Maryland 20706
}

\begin{abstract}
We present data on the vulnerability of a variety of candidate spacecraft electronics to proton and heavy-ion induced single-event effects and proton-induced damage. We also present data on the susceptibility of parts to functional degradation resulting from total ionizing dose at low dose rates (0.003-0.33 $\operatorname{Rads}(\mathrm{Si}) / \mathrm{s})$. Devices tested include optoelectronics, digital, analog, linear bipolar, hybrid devices, Analog to Digital Converters (ADCs), Digital to Analog Converters (DACs), and DC-DC converters, among others.
\end{abstract}

\section{INTRODUCTION}

Recent experience in satellite design has shown a continuation, if not an acceleration, of the trend toward the use of commercial parts. As spacecraft designers use increasing numbers of commercial and emerging technology devices to meet stringent performance, economic and schedule requirements, ground-based testing of such devices for susceptibility to single-event effects (SEE), Co-60 total ionizing dose (TID) and proton-induced damage has assumed ever greater importance. Recent experience in satellite design has also emphasized the increased susceptibility of bipolar devices to damage from Total Ionizing Dose (TID) at lowdose-rates (0.001-0.01 Rads' $(\mathrm{Si}) / \mathrm{s})$. This paper discusses the results of low-dose-rate (0.003-0.33 Rads(Si)/s) testing of a variety of devices representing several different vendors and fabricated in many different technologies.

The studies discussed here were undertaken to establish the sensitivities of candidate spacecraft electronics to heavyion and proton induced single-cvent upsets (SEU), singleevent latchup (SEL), single event transient (SET), TID, and proton damage (ionizing and non-ionizing).

\section{TEST TECHNIQUES AND SETUP}

\section{A. Test Facilities}

All SEE and proton-induced damage tests were performed between February 1999) and February 2000. TID tests were performed between February 1998 and February 2000. TID testing was performed using a $\mathrm{Co}^{60}$ source at the Goddard Space Flight Center Radiation Effects Facility (GSFC REF). Heavy-Ion experiments were conducted at the Brookhaven National Laboratories Single-Event Upset Test Facility (SEUTF). The SEUTF uses a Tandem Van De Graaf accelerator that can provide ions and energies suitable for SEU tcsting. Test boards containing the device under test (DUT) were mounted within a vacuum chamber. The DUT was irradiated with ions with linear energy transfers (LETs) of 1.1 to $120 \mathrm{McV} \cdot \mathrm{cm}^{2} \mathrm{mg}^{-1}$, with fluences from $1 \times 10^{5}$ to $1 \times 10^{7}$ particles $\mathrm{cm}^{-2}$. Fluxes ranged from $1 \times 10^{2}$ to $1 \times 10^{5}$ particles $\mathrm{cm}^{-2}$ per second, depending on the device sensitivity. The ions used are listed in Table 1. LETs between the values listed could be obtained by changing the angle of incidence of ion beam onto the DUT, thus changing the path length of the ion through the DUT. Energies and LETs available varied slightly from one test date to another.

Proton SEE and damage tests were performed at four facilitics: the University of California Davis (UCD) Crocker Nuclear Laboraton: (CNL), TRI-University Meson Facility (TRIUMF), and the Indiana University Cyclotron Facility (IUCF). Proton test energies are listed in Table 2. Typically, the DUT was irradiated to a fluence from $1 \times 10^{10}$ to $1 \times 10^{11}$ particles $\mathrm{cm}^{-2}$, wilh fluxes of $1 \times 10^{8}$ particles ${ }^{\cdot} \mathrm{cm}^{-2}$ per second. 

Table I: BNL T'est I leavy lons

\begin{tabular}{|l|c|c|c|}
\hline Ion & $\begin{array}{c}\text { Energy, } \\
\mathrm{MeV}\end{array}$ & $\begin{array}{c}\text { LET in Si, } \\
\mathrm{MeV} \cdot \mathrm{cm}^{2} / \mathrm{mg}\end{array}$ & $\begin{array}{c}\text { Range in } \\
\mathrm{Si} \text { micronmeter s }\end{array}$ \\
\hline $\mathrm{C}^{12}$ & 102 & 1.4 & 193 \\
\hline $\mathrm{F}^{19}$ & 141 & 3.4 & 126 \\
\hline $\mathrm{Si}^{35}$ & 186 & 7.9 & 85.3 \\
\hline $\mathrm{Cl}^{35}$ & 210 & 11.4 & 65.8 \\
\hline $\mathrm{Ti}^{48}$ & 227 & 18.8 & 47.5 \\
\hline $\mathrm{Ni}^{58}$ & 266 & 26.6 & 41.9 \\
\hline $\mathrm{Br}^{79}$ & 290 & 37.2 & 39 \\
\hline $\mathrm{I}^{127}$ & 320 & 59.7 & 34 \\
\hline $\mathrm{Au}^{197}$ & 350 & 82.3 & 27.9 \\
\hline
\end{tabular}

Table 2: Proton Test Facilities

\begin{tabular}{|l|c|c|}
\hline \multicolumn{1}{|c|}{ Facility } & Particle & $\begin{array}{c}\text { Particle } \\
\text { Energy, } \\
\text { (MeV) }\end{array}$ \\
\hline $\begin{array}{l}\text { University of California at Davis (UCD) } \\
\text { Crocker Nuclear Laboratory }\end{array}$ & Proton & $26.6-63$ \\
\hline TRI-University Meson Facility (TRIUMF) & Proton & $50-500$ \\
\hline $\begin{array}{l}\text { Indiana University Cyclotron Facility } \\
\text { (IUCF) }\end{array}$ & Proton & $54-197$ \\
\hline
\end{tabular}

\section{B. Test Method}

Depending on the DUT and the test objectives, one or more of three SEE test methods were used:

Dynamic - the DUT was exercised continuously while being exposed to the beam, and the errors were counted, generally by comparing DUT output to a unirradiated reference device or other expected output. Different device modes and clock speed may affect SEE results.

Static - the DUT was loaded prior to irradiation; data was retrieved and errors were counted after irradiation.

Biased (SEL only) - the DUT was biased and clocked while ICC (power consumption) was monitored for SEL or other destructive effects.

In SEE experiments, DUTs were monitored for soft errors, such as SEUs and for hard errors, such as SEL. Detailed descriptions of the types of errors observed are noted in the individual test results.

Proton damage tests were performed on biased devices with functionality and parametrics being measured either continually during irradiation or after step irradiations (for example, every $10 \mathrm{krad}(\mathrm{Si})$ ).

TID testing was performed using a Co-60 source at the Radiation Effects Facility at Goddard Space Flight Center. The source is capable of delivering dose rates from 0.003-1.1 $\operatorname{Rad}(\mathrm{Si}) / \mathrm{s}$, with dosimetry being performed by an ion chamber probe. TID testing used method 1019.5 as a guide.

Pre-irradiation electrical characterization was undertaken on all controls and test devices by means of functional and parametric tests. The parts were then irradiated in steps from
1 to $20 \mathrm{kRad}(\mathrm{Si})$ and tested after each step for parametric degradation and functionality.

Displacement damage test guidelines are currently under development. Optocoupler characterization approaches used in this study are found in $\{2\}$.

Unless otherwise noted, all tests were performed at room temperature and nominal power supply voltages.

\section{TISTI RISULTSS OVERVIEW}

SEE test results are summarized in Table 3. Displacement damage effects test results are summarized in Table 4 . Abbreviations for principal investigators are listed in Table 5 . TID test results are summarized in Table 6. This paper is a summary of results. Complete test reports are available online at hitp://radhome.gsfc.nasa.gov [1].

These tables use the following abbreviations and conventions:

$\mathrm{H}=$ heavy ion test
$\mathrm{P}=$ proton test $(\mathrm{SEE})$
$\mathrm{LET}=$ linear energy transfer $\left(\mathrm{MeV} \cdot \mathrm{cm}^{2} / \mathrm{mg}\right)$

LET $_{\text {th }}=$ lincar energy transfer threshold (the minimum LET valuc to cause an effect at a fluence of $1 \times 10^{7}$ particles $/ \mathrm{cm}^{2}$ ) in $\mathrm{MeV} \cdot \mathrm{cm}^{2} / \mathrm{mg}$

LET max $=$ highest tested LET

SEU $=$ single event upset

$\mathrm{SEL}=$ single event latchup

$\mathrm{SET}=$ single event transient

$\mathrm{DD}=$ displacement damage

$<=$ SEE observed at lowest tested LET

$>=$ No SEE obseried at highest tested LET

$\mathrm{PD}=$ proton damagc (both ionizing and non-ionizing)

$\mathrm{TID}=$ total ionizing dose

$\sigma=$ cross section $\left(\mathrm{cm}^{2} /\right.$ device, unless specified as $\left.\mathrm{cm}^{2} / \mathrm{bit}\right)$

$\sigma_{\text {SAT }}=$ saluration cross section $\left(\mathrm{cm}^{2} /\right.$ device, unless specified as $\mathrm{cm}^{2} / \mathrm{bit}$ )

$\mathrm{LDC}=$ lot date code

VOS $=$ offset voltage

Vrms = root mean squared voltage

$I_{S S}=V_{S S}$ current

$I_{D D}=V_{D D}$ current

$I_{\mathrm{L}}=$ low current

$\mathrm{I}_{\mathrm{tH}}=$ high current

$I_{\text {COFF }}=V_{\text {DO }}$ current ofr

$I_{S O F F}=V_{S S}$ current off

$\mathrm{R}_{\mathrm{N}}$ or $\mathrm{RDS}_{\mathrm{iN}}=$ resistance

VGS = gate source voltage

VDS = drain source voltage

Unless ollerwise noted. all $\mathrm{LET}_{\mathrm{th}} \mathrm{S}$ are in $\left(\mathrm{MeV} \cdot \mathrm{cm}^{2} / \mathrm{mg}\right)$ and all cross sections are in $\mathrm{cm}^{2} /$ device 
Table 3: Summary of Slil: Test Results

\begin{tabular}{|c|c|c|c|c|c|}
\hline Part Number & Function & Manufacturer & $\begin{array}{l}\text { Particle: } \\
\text { (Facility)P.I. }\end{array}$ & $\begin{array}{c}\text { Testing } \\
\text { Preformed }\end{array}$ & Summary of Results \\
\hline \multicolumn{6}{|l|}{ Power Devices: } \\
\hline TL7702B & Power Supervisor & $\begin{array}{l}\text { Texas } \\
\text { Instruments }\end{array}$ & $\begin{array}{l}H:(B N L) R R \\
P:(I U C F) K L\end{array}$ & $\begin{array}{l}\text { SEE and } \\
P: \text { SEE }\end{array}$ & $\begin{array}{l}\text { H: SEL LET } \text { ih }>73, \text { SET LET } \\
\sigma_{\text {in }}=5 \times 10^{-5} \text {, results application specific } \\
\text { P: No SEU, SETs observed }\end{array}$ \\
\hline TL7705B & Power Supervisor & $\begin{array}{l}\text { Texas } \\
\text { Instruments }\end{array}$ & $\begin{array}{l}H:(B N L) R R \\
P:(I U C F) K L\end{array}$ & $\begin{array}{l}\text { SEE and } \\
P \text { : SEE }\end{array}$ & $\begin{array}{l}\text { H: SEL LET in }>73, \text { SET LET in } \sim 2.7-3.3 \\
\sigma_{\text {SAT }}=1 \times 10^{-4} \text {, results application specific } \\
\text { P: No SEU, SETs observed }\end{array}$ \\
\hline TL7770-5 & Power Supervisor & $\begin{array}{l}\text { Texas } \\
\text { Instruments }\end{array}$ & $H:(B N L) R R$ & SEE & $H:$ SEL LET th $>73$, SET observed but not measured \\
\hline TLC7705 & Power Supervisor & $\begin{array}{l}\text { Texas } \\
\text { Instruments }\end{array}$ & $\mathrm{H}:(\mathrm{BNL}) \mathrm{RR}$ & SEE & $\begin{array}{r}\text { H: SEL LET } \\
\sigma_{\text {SAT }}>1.5 \times 10^{-4}, \text { results application specific }\end{array}$ \\
\hline \multicolumn{6}{|l|}{ ADC/DAC: } \\
\hline MX7225UQ & 8 bit DAC & Maxim & $H:(B N L) R R$ & SEL & SEL LET th $>90$ \\
\hline AD571S & AD Converter & $A D$ & $H:(B N L) R R / J H$ & SEL & SEL LET $T_{\text {in }}>60$ \\
\hline LTC1419 & AND Converter & Linear Tech & $\mathrm{H}:(B N L) R R / J H$ & SEL & SEL LET $T_{\text {th }}>60$ \\
\hline DACO8 & $\begin{array}{l}\text { Digital to Analog } \\
\text { Converter }\end{array}$ & $A D$ & $\mathrm{H}:(B N L) \mathrm{SH}$ & SELSEU & $\begin{array}{l}\text { SEL LET } T_{\text {in }}>60, \text { maybe SEU's at LET }=60 \text { and }+/-15 \mathrm{~V} \\
\text { and FF input, SEU/SEL LET th }>119.6 \text { (application } \\
\text { Specific) }\end{array}$ \\
\hline \multicolumn{6}{|r|}{ 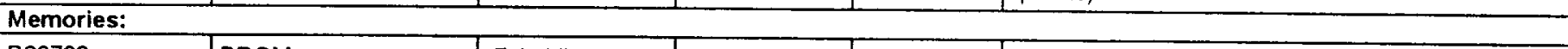 } \\
\hline R29793 & PROM & Fairchild & $\mathrm{H}:(\mathrm{BNL}) \mathrm{JH}$ & SEE & $\begin{array}{l}\text { SEL LET } \\
\text { SEU }>37.4, \text { two types of SEUs were observed, } \\
\text { Iin }-3, \sigma_{S A T}=3 \times 10^{-3}\end{array}$ \\
\hline \multicolumn{6}{|c|}{ Digital Signal Processors: } \\
\hline $\mathrm{RH} 21020$ & DSP & Lockheed-Martin & P: (UCD)KL & P: SEE & $\sigma=7.64 \times 10^{.11}$ \\
\hline TSC21020F & DSP & $\begin{array}{l}\text { Temic } \\
\text { Semiconductor }\end{array}$ & P: (UCD)KL & P: SEE & $\sigma=9.9 \times 10^{-13}$ \\
\hline \multicolumn{6}{|l|}{ Logic Devices: } \\
\hline 10502 & ECL Multiple NOR Gate & Motorola & $H:(B N L) J H$ & SELISEU & $\begin{array}{l}\text { SEL LET in }>60, \text { SEUs with LET } \\
\sigma_{\text {SAT }} \sim-26 \text { and } \\
\end{array}$ \\
\hline MC74LCX08 & 2 INP and Gate & Motorola & $H:(B N L) R R / J H$ & SEL & SEL LET $T_{\text {th }}>=60$ \\
\hline \multicolumn{6}{|c|}{ Fiber Optic Links: } \\
\hline HFBR-53D5 & Transceiver & $\mathrm{HP}$ & $P:(U C D) P M$ & $P: S E E$ & $\begin{array}{l}\text { Proton induced SETs observed, No destructive } \\
\text { conditions observed to } \sim 25 \mathrm{krads}(\mathrm{Si}) \text { of } 63 \mathrm{MeV} \\
\text { protons }\end{array}$ \\
\hline TTC-155M4 & Transceiver & Lasermate & $P:(U C D) P M$ & P: SEE & $\begin{array}{l}\text { Proton induced SETs observed, No destructive } \\
\text { conditions observed to } \sim 25 \mathrm{krads} \text { (Si) of } 63 \mathrm{MeV} \\
\text { protons }\end{array}$ \\
\hline TTC-155M2 & Transceiver & Lasermate & $P:(U C D) P M$ & P: SEE & $\begin{array}{l}\text { Proton induced SETs observed, No destructive } \\
\text { conditions observed to }-25 \mathrm{krads}(\mathrm{Si} \text { ) of } 63 \mathrm{MeV} \\
\text { protons }\end{array}$ \\
\hline \multicolumn{6}{|c|}{ Linear Bipolar Devices: } \\
\hline CLC 449 & OP AMP & $\begin{array}{l}\text { National } \\
\text { Semiconductor }\end{array}$ & $H:(B N L) R R$ & SET; SEL & $\begin{array}{l}\text { No SEL or SET to LETmax }=60 ; \text { SET result is } \\
\text { application specific }\end{array}$ \\
\hline PA07 & High Power OP AMP & APEX & $H:(B N L) R R$ & SET; SEL & $\begin{array}{l}\text { SEL and SET are application specific. No SEL or SET } \\
\text { to LETmax }=37\end{array}$ \\
\hline LMC6081 & Precision OP AMP & $\begin{array}{l}\text { National } \\
\text { Semiconductor }\end{array}$ & $\mathrm{H}:(\mathrm{BNL}) \mathrm{JH}$ & SEL & No SEL to LETmax $=60$ \\
\hline HS139 & Comparator & Harris & $H:(B N L) J H$ & SET; SEL & SETs observed, No SELs observed \\
\hline LM139 & Comparator & $\begin{array}{l}\text { National } \\
\text { Semiconductor }\end{array}$ & $\begin{array}{l}P:(U C O) J H \\
H:(B N L) J H\end{array}$ & $\begin{array}{l}\text { P:SET } \\
\text { HI: SET; } \\
\text { SEL }\end{array}$ & $\begin{array}{l}\text { No SETS observed with test setup used; SET } \\
\text { LET } T_{\text {in }}=20 ; \sigma_{\text {SAT }}=2 \times 10^{-4} \\
\text { SEL LET }>59.8\end{array}$ \\
\hline MAX962 & Comparator & Analog Devices & $\mathrm{H}:(\mathrm{BNL}) \mathrm{JH}$ & $\overline{S E L}$ & NO SEL IO LETMax $=60$ \\
\hline
\end{tabular}




\begin{tabular}{|c|c|c|c|c|c|}
\hline Part Number & Function & Manufacturer & $\begin{array}{l}\text { Particle: } \\
\text { (Facility)P.I. }\end{array}$ & $\begin{array}{l}\text { Testing } \\
\text { Preformed }\end{array}$ & Surnmary of Results \\
\hline \multicolumn{6}{|c|}{ Linear Bipolar Devices (Cont.): } \\
\hline AD783SQ & $\begin{array}{l}\text { Sample and Hold } \\
\text { Amplifier }\end{array}$ & Analog Devices & $\mathrm{H}:(\mathrm{BNL}) \mathrm{RR}$ & SET; SEL & SEL LET $T_{\text {in }}>90$, SET LET th $\sim 7, \sigma_{\text {SAT }}=1 \times 10^{-4} \mathrm{~cm}^{2}$ \\
\hline A250 & Charge Sensitive Amp & Amptek & $H:(B N L) R R$ & SET:SEL & $\begin{array}{l}\text { No SEL or SET to LETmax }=60 ; \text { SET is application } \\
\text { specific }\end{array}$ \\
\hline MSA0670 & MMIC Amplifier & HP & $H:(B N L) J H$ & SET; SEL & No SEL or SET to LET $\max =85$ \\
\hline \multicolumn{6}{|l|}{ Optocouplers: } \\
\hline OLH5601 & Optocoupler & Isolink & $P:(T R I U M F) R R$ & SET & $\begin{array}{l}\text { Proton induced transients were observed, no } \\
\text { significant degradation }\end{array}$ \\
\hline $6 \mathrm{~N} 134$ & Optocoupler & Micropac & $P:(T R I U M F) R R$ & SET & $\begin{array}{l}\text { Proton induced transients were observed, no } \\
\text { significant degradation }\end{array}$ \\
\hline \multicolumn{6}{|l|}{ Others: } \\
\hline DS1670E & System Controller & $\begin{array}{l}\text { Dallas } \\
\text { Semiconductor }\end{array}$ & H: (BNL)RR & SEL & SEL LET Th -15 \\
\hline SN54LVTH16244A & Buffer/Drivers & $\begin{array}{l}\text { Texas } \\
\text { instruments }\end{array}$ & $H:(B N L) R R / J H$ & SEL & No SEL to LET $\max =60$ \\
\hline CGS74LCT2524 & Clock Driver & $\begin{array}{l}\text { National } \\
\text { Semiconductor }\end{array}$ & $\mathrm{H}:(\mathrm{BNL}) \mathrm{RR} / \mathrm{JH}$ & SEL & No SEL to LETmax $=60$ \\
\hline $\mathrm{MIC} 4423$ & MOSFET driver & Micrel & $H:(B N L) R R$ & SET; SEL & $\begin{array}{l}\text { No SEL or SET to LETmax }=60 ; \mathrm{SEL} \text { and SET are } \\
\text { application specific }\end{array}$ \\
\hline MC74HC4538A & Multivibrator & Motorola & $\mathrm{H}:(\mathrm{BNL}) \mathrm{RR} / \mathrm{JH}$ & SEL & No SEL to LETmax $=60$ \\
\hline DS1803 & $\begin{array}{l}\text { Dual Digital } \\
\text { Potentiometer }\end{array}$ & $\begin{array}{l}\text { Dallas } \\
\text { Semiconductor }\end{array}$ & $H:(B N L) R R$ & SEL; SEU & LET $_{\text {Ih }}>20 ;$ SEL observed at LET $<37$ \\
\hline
\end{tabular}

Table 4: Summary of Displacement Damage Test Results

\begin{tabular}{|c|c|c|c|c|c|}
\hline Part Number & Function & Manufacturer & $\begin{array}{c}\text { Particle: } \\
\text { (Facility)P.I. }\end{array}$ & $\begin{array}{l}\text { Testing } \\
\text { Preformed }\end{array}$ & Summary of Results \\
\hline \multicolumn{6}{|c|}{ Linears Bipolar Devices: } \\
\hline LM111 & Comparator & $\begin{array}{l}\text { National } \\
\text { Semiconductor }\end{array}$ & $\begin{array}{r}P:(\cup C D) J H \\
\text { (IUCF) JH }\end{array}$ & DD & Saw enhanced damage from Protons vs Co- 60 \\
\hline \multicolumn{6}{|l|}{ Optocouplers: } \\
\hline OLH249 & Optocoupler & Isolink & $P:(U C D) K L$ & DD & CTR degradation observed. See [2] \\
\hline 66099 & Optocoupler & Micropac & $P:(U C D) R R$ & $P: D D$ & CTR measurements for $63 \mathrm{MeV} ; \mathrm{Vce}=5 \mathrm{~V}$ \\
\hline \multicolumn{6}{|l|}{ Optoelectronics: } \\
\hline 00800 & LED & Optodiode & P: (UCD)RR/PM & DD & $60 \%$ light output degradation at $2.7 \times 10^{10}$ \\
\hline$H F E-4080$ & $\begin{array}{l}\text { Linear feedback shift } \\
\text { register }\end{array}$ & Honeywell & $P:(U C D) C M$ & DD & \\
\hline
\end{tabular}

Table 5: List of Principal Investigators

\begin{tabular}{|l|c|}
\hline \multicolumn{1}{|c|}{ Principal Investigator (PI) } & Abbreviation \\
\hline Kenneth LaBel & $\mathrm{KL}$ \\
\hline Robert Reed & $\mathrm{RR}$ \\
\hline Jim Howard & $\mathrm{JH}$ \\
\hline Paul Marshall & $\mathrm{PM}$ \\
\hline Cheryl Marshall & $\mathrm{CM}$ \\
\hline
\end{tabular}


Table Ga: Summary of NASA GSIC TII) Test Results - Comparator

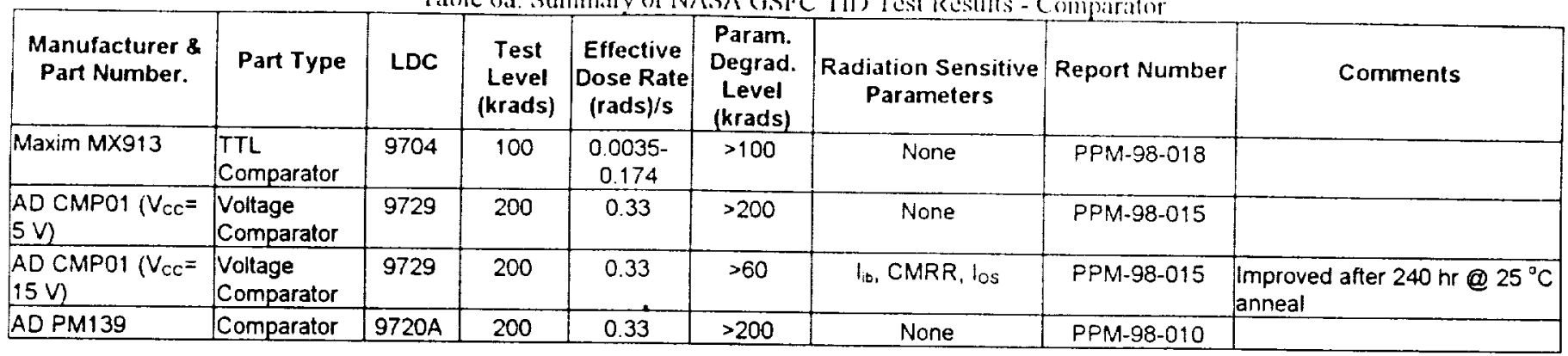

Table 6b: Summary of NASA GSFC TID Test Resuls - Operational Amplitiers

\begin{tabular}{|c|c|c|c|c|c|c|c|c|}
\hline $\begin{array}{c}\text { Manufacturer \& } \\
\text { Part Number. }\end{array}$ & Part Type & LDC & $\begin{array}{c}\text { Test } \\
\text { Level } \\
\text { (krads) }\end{array}$ & $\begin{array}{c}\text { Effective } \\
\text { Dose Rate } \\
\text { (rads)/s }\end{array}$ & $\begin{array}{c}\text { Param. } \\
\text { Degrad. } \\
\text { Level } \\
\text { (krads) }\end{array}$ & $\begin{array}{c}\text { Radiation Sensitive } \\
\text { Parameters }\end{array}$ & Report Number & Comments \\
\hline NSC LMC6464 & \begin{tabular}{|l|} 
CMOS Op \\
Amp \\
\end{tabular} & 9722 & $5.0,10.0$ & 0.03 & N/A & Multiple parameters & PPM-99-041 & $\begin{array}{l}\text { Catastrophic failure at 5-10 } \\
\operatorname{krads}(\mathrm{Si})\end{array}$ \\
\hline $\begin{array}{l}\text { NSC (Comlinear) } \\
\text { CLC502 } \\
\end{array}$ & Op Amp & $\begin{array}{c}\text { Not } \\
\text { marked }\end{array}$ & $5-100$ & $\begin{array}{l}0.035 \\
0.174 \\
\end{array}$ & 100 & N/A & PPM-98-018 & \\
\hline AD AD783SQ & $\begin{array}{l}\text { Sample \& } \\
\text { Hold Amp. }\end{array}$ & 9702 & $2.5-50$ & 0.04 & N/A & $N / A$ & PPM-99-040 & $\begin{array}{l}\text { Passed all tests to } 50 \\
\text { krads }(\mathrm{Si})\end{array}$ \\
\hline$A D$ AD620 & Inst. Op Amp & 9815 & $5.0-25$ & 0.04 & $5-10$ & Bias currents & PPM-99-029 & \\
\hline$A D A D 845$ & $\begin{array}{l}\text { CBFET OP } \\
\text { Amp }\end{array}$ & 9846 & $2.5-50$ & 0.04 & $>20$ & Multiple parameters & PPM-99-026 & \\
\hline AD OP-07 & Op Amp & 9723B & $10-40$ & 0.14 & \begin{tabular}{c|}
$>10$ \\
$\operatorname{krads}(\mathrm{Si})$ \\
\end{tabular} & Offset voltage & PPM-99-017 & Some recovery after anneal \\
\hline AD OP-O7 & Op Amp & $9724 \mathrm{~A}$ & $5-40$ & 0.14 & -20 & Offset voltage & PPM-99-016 & Some recovery after anneal \\
\hline$A D O P-07$ & Op Amp & 9724 & $20-200$ & $\begin{array}{c}0.14 \text { and } \\
0.58 \\
\end{array}$ & -20 & Multiple parameters & PPM-99-001 & $\begin{array}{l}\text { Degradation worse for higher } \\
\text { rate. Lot had outliers in Vos }\end{array}$ \\
\hline$A D O P-07$ & Op Amp & 9723B & $20-200$ & 0.33 & -20 & $\begin{array}{l}\text { Offset voltage, then } \\
\text { multiple parameters }\end{array}$ & PPM-98-011 & Lot had outliers in Vos. \\
\hline AD AMPO1 & Inst. Amp. & $9818 \mathrm{~A}$ & $2.5-50$ & 0.02 & $>5$ & \begin{tabular}{|c|}
$\begin{array}{c}\text { Offset voltages, gain } \\
\text { errors }\end{array}$ \\
\end{tabular} & PPM-99-015 & \\
\hline AD OP467 & Op. Amp. & $9812 \mathrm{~A}$ & $2.5-50$ & 0.04 & $>10$ & Bias currents & PPM-99-004 & \\
\hline$A D O P 400$ & Op. Amp & $9814 A$ & $2.5-50$ & 0.04 & $>5$ & $\begin{array}{l}\text { Bias currents, slew } \\
\text { rate, gain }\end{array}$ & PPM-99-003 & \\
\hline AD OP270 & Op. Amp. & 9815 & $2.5-50$ & 0.04 & $>5$ & Bias currents & PPM-98-029 & \\
\hline$A D O P 27$ & Op. Amp. & 9721A & $20-200$ & 0.333 & $<20-40$ & Offset voltages & PPM-98-009 & \\
\hline$A D O P 15$ & Op. Amp. & $9722 \mathrm{~A}$ & $20-200$ & 0.333 & $20-40$ & $\begin{array}{l}\text { Offset voltage, } \\
\text { leakage currents }\end{array}$ & PPM-98-008 & \\
\hline AD AD585 & $\begin{array}{l}\text { Sample } \\
\text { \&Hold Amp. }\end{array}$ & 9648 & $5-100$ & $\begin{array}{l}0.035 \\
0.174\end{array}$ & $>30$ & $\begin{array}{c}\text { Common-mode } \\
\text { rejection ratio, offset } \\
\text { voltage }\end{array}$ & PPM-98-006 & \\
\hline AD AD524 & Inst. Amp. & $9650 \mathrm{~A}$ & $5-100$ & 0.072 & $>20$ & Offset voltages & PPM-98-005 & \\
\hline LT LF155A & $\begin{array}{l}\text { Op. Amp. } \\
\text { JFET input }\end{array}$ & 9811 & $2.5-30$ & 0.04 & $>10$ & Bias currents & PPM-99-035 & \\
\hline LT LT1010 & Pwr. Buffer & 9808 & $2.5-100$ & 0.08 & $>100$ & N/A & PPM-99-010 & \\
\hline LT LF198 & $\begin{array}{l}\text { Sample \& } \\
\text { Hold Amp. }\end{array}$ & 9129 & $20-200$ & 0.33 & $>200$ & $\mathrm{~N} / \mathrm{A}$ & PPM-99-009 & \\
\hline LT LF147 & Op. Amp. & 9803 & $\begin{array}{l}2.0-10 \\
\text { (low- } \\
\text { dose } \\
\text { rate) } \\
2.5-50 \\
\end{array}$ & $\begin{array}{l}0.004 \\
0.02\end{array}$ & $\begin{array}{c}\text { See in } \\
\text { comments } \\
\text { section }\end{array}$ & Multiple parameters & PFM-99-002 & $\begin{array}{l}\text { Low-rate tests, no significant } \\
\text { degradation to } 10 \mathrm{krads}(\mathrm{Si}) \\
\text { * High rate: } 5 \mathrm{krads} \text { showed } \\
\text { sig. Degradation; not seen at } \\
\text { lower rate } \\
\end{array}$ \\
\hline $\begin{array}{l}\text { Burr-Brown } \\
\text { INA117SM } \\
\end{array}$ & Diff. Amp. & 9837 & $10-50$ & 0.06 & $>17.5$ & Offset voltage & PPM-99-033 & \\
\hline Apex PA07M & $\begin{array}{l}\text { Power Op. } \\
\text { Amp. } \\
\end{array}$ & 9918 & $10-50$ & 0.06 & $>17.5$ & Offset voltage & PPM-99-032 & \\
\hline Amplek A250 & Preamplifier & 9902 & $10-100$ & 0.07 & $>100$ & $N / A$ & PPM-99-031 & \\
\hline Maxim MAX494 & Op. Amp. & 9639 & $5-100$ & 0.06 & $>10$ & Bias currents & PPM-98-019 & \\
\hline $\begin{array}{l}\text { Omnirel } \\
\text { OM11725SMX }\end{array}$ & Op. Amp. & 9735 & $5-75$ & 0.043 & $\begin{array}{c}>20 \\
\operatorname{krads}(S i)^{*}\end{array}$ & Line voltages & PPM-98-002 & $\begin{array}{l}\text { Note: catastrophic functional } \\
\text { failure } 15-75 \mathrm{krads}(\mathrm{Si})\end{array}$ \\
\hline
\end{tabular}


Table 6e: Summary of NASA GSl:C l11) Test Results - Analog-to-1)igital and I)igital-to-Analog Converters

\begin{tabular}{|c|c|c|c|c|c|c|c|c|}
\hline $\begin{array}{l}\text { Manufacturer \& } \\
\text { Part Number. }\end{array}$ & Part Type & LDC & $\begin{array}{c}\text { Test } \\
\text { Level } \\
\text { (krads) }\end{array}$ & $\begin{array}{c}\text { Effective } \\
\text { Dose Rate } \\
\text { (rads)/s }\end{array}$ & $\begin{array}{c}\text { Param. } \\
\text { Degrad. } \\
\text { Level } \\
\text { (krads) }\end{array}$ & $\begin{array}{c}\text { Radiation Sensitive } \\
\text { Parameters }\end{array}$ & Report Number & Comments \\
\hline AD AD7821 & 8-Bit ADC & 9727 & $2.5-50$ & 0.04 & $>30$ & $\begin{array}{l}\text { Missing codes, } \\
\text { integral nonlinearity }\end{array}$ & PPM-99-045 & \\
\hline AD AD7885 & 16-Bit ADC & 9827 & $2.5-50$ & 0.06 & $>50$ & $N / A$ & PPM-99-039 & \\
\hline AD AD571 & 10-Bit ADC & 9746 & $20-200$ & 0.33 & $\geq 200$ & N/A & PPM-98-024 & \\
\hline AD AD976 & $\begin{array}{l}\text { 16-Bit ADC } \\
\text { (BiCMOS) }\end{array}$ & 9723 & $5-100$ & 0.033 & $<5$ & $\begin{array}{l}\text { Missing codes, } \\
\text { integral and } \\
\text { differential } \\
\text { nonlinearity }\end{array}$ & PPM-98-001 & $\begin{array}{l}\text { Note parts may exhibit low- } \\
\text { dose-rate susceptibility }\end{array}$ \\
\hline AD DACOB & 8-Bit DAC & 9831 & $10-50$ & 0.05 & $>25$ & $\begin{array}{c}\text { Power supply } \\
\text { sensitivity }\end{array}$ & PPM-99-036 & \\
\hline AD AD7535 & 14-Bit DAC & 9812 & $10-25$ & 0.05 & $>10$ & $\begin{array}{l}\text { Integral and } \\
\text { differential } \\
\text { nonlinearity }\end{array}$ & PPM-99-027 & \\
\hline AD AD7545 & 12-Bit DAC & 9807 & $2.5-30$ & 0.07 & $>5$ & $\begin{array}{c}\begin{array}{c}\text { Integral and } \\
\text { differential } \\
\text { nonlinearity; leakage } \\
\text { currents }\end{array} \\
\end{array}$ & PPM-99-022 & \\
\hline$\widehat{A D ~ A D 8222 ~}$ & 12-Bit DAC & 9738 & $\begin{array}{c}\text { GroupA: } \\
2.5-5 ; \\
\text { Group B: } \\
1-10 \\
\end{array}$ & $\begin{array}{c}\text { Group A: } \\
0.004 \\
\text { Group B: } \\
0.003\end{array}$ & $\begin{array}{c}A:<2.5 \\
B:<1\end{array}$ & Multiple parameters & PPM-99-006 & \\
\hline Maxim MX7225 & 8-Bit DAC & 9321 & $2.5-30$ & 0.04 & $>10$ & Multiple parameters & PPM-99-042 & \\
\hline Maxim MX536 & $\begin{array}{l}\text { RMS-DC } \\
\text { Converter }\end{array}$ & 9817 & $2.5-100$ & 0.06 & $>10$ & VOS, Vrms & PPM-99-0C8 & \\
\hline $\begin{array}{l}\text { Micronetworks } \\
\text { MN5295 }\end{array}$ & 16-bit DAC & $\begin{array}{l}9549 \\
9540\end{array}$ & $5-35$ & 0.019 & $>15$ & $\begin{array}{l}\text { Missing codes, } \\
\text { integral and diff. } \\
\text { Nonlinearity }\end{array}$ & PPM-99-018 & \\
\hline
\end{tabular}

Table 6d: Summary of NASA GSFC TID Test Results - Voltage Regulators and Voltage References

\begin{tabular}{|c|c|c|c|c|c|c|c|c|}
\hline $\begin{array}{l}\text { Manufacturer \& } \\
\text { Part Number. }\end{array}$ & Part Type & LDC & $\begin{array}{c}\text { Test } \\
\text { Level } \\
\text { (krads) }\end{array}$ & $\begin{array}{c}\text { Effective } \\
\text { Dose Rate } \\
\text { (rads)/s }\end{array}$ & $\begin{array}{c}\text { Param. } \\
\text { Degrad. } \\
\text { Level } \\
\text { (krads) }\end{array}$ & $\begin{array}{l}\text { Radiation Sensitive } \\
\text { Parameters }\end{array}$ & Report Number & Comments \\
\hline Linfinity PIC7527 & $\begin{array}{l}\text { Switching } \\
\text { Regulator }\end{array}$ & 9450 & $2.5-50$ & 0.06 & $>50$ & N/A & PPM-99-038 & \\
\hline NSC LM117K & $\begin{array}{l}\text { Voltage } \\
\text { Regulator }\end{array}$ & 9808 & $10-30$ & 0.08 & $>10$ & Line voltages & PPM-99-034 & \\
\hline NSC LM117H & \begin{tabular}{|l|} 
Voltage \\
Regulator
\end{tabular} & 9727 & $20-200$ & 0.33 & $>20$ & Line voltages & PPM-98-026 & No improvement after anneal \\
\hline NSC LM117HVK & $\begin{array}{l}\text { Voltage } \\
\text { Regulator }\end{array}$ & 9732 & $20-200$ & 0.33 & $>/ \sim 20$ & Line voltages & PPM-98-021 & \\
\hline NSC LM117HVH & $\begin{array}{l}\text { Voltage } \\
\text { Regulator }\end{array}$ & 9727 & $20-200$ & 0.33 & $>20$ & Line voltages & PPM-98-020 & \\
\hline Omnirel OM3914 & \begin{tabular}{|l|} 
Neg. Voltage \\
Reg.
\end{tabular} & 9909 & $2.5-50$ & 0.05 & $>10$ & $\begin{array}{c}\text { Ref. Voltage, line } \\
\text { reg. }\end{array}$ & PPM-99-028 & \\
\hline \begin{tabular}{|l|} 
Omnirel \\
OM1850STM3
\end{tabular} & \begin{tabular}{|l|} 
Voltage \\
Regulator \\
\end{tabular} & 9912 & $2.5-50$ & 0.04 & $>10$ & $\begin{array}{c}\text { Ref. Voltage, line } \\
\text { reg. }\end{array}$ & PPM-99-024 & \\
\hline AD AD588 & $\begin{array}{l}\text { Voltage } \\
\text { Reference }\end{array}$ & 9814 & $2.5-100$ & 0.05 & $>15$ & $\begin{array}{l}\text { Minor shifts in } \\
\text { voltage levels }\end{array}$ & PPM-99-014 & \\
\hline$A D A D 780$ & \begin{tabular}{|l} 
Voltage \\
Reference
\end{tabular} & 9728 & $2.5-100$ & 0.08 & $>100$ & N/A & PPM-99-011 & \\
\hline
\end{tabular}




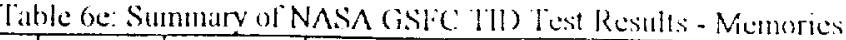

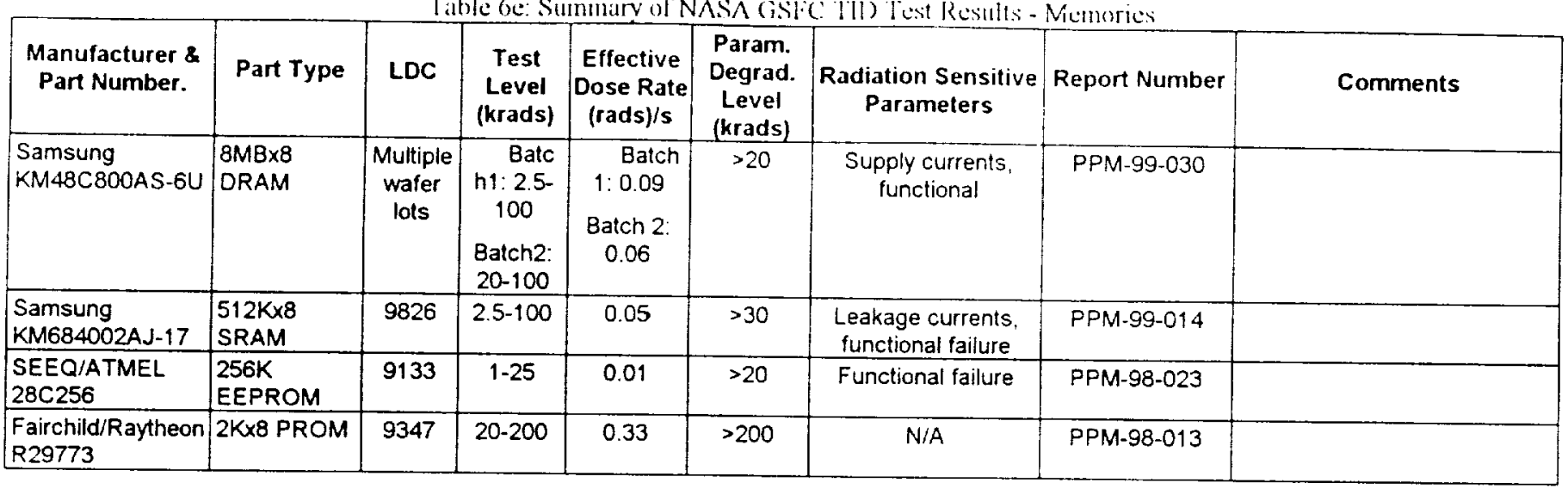

Table 6f: Summary of NASA GSFC TID Test Results - Analog Switches and Multiplexers

\begin{tabular}{|c|c|c|c|c|c|c|c|c|}
\hline $\begin{array}{l}\text { Manufacturer \& } \\
\text { Part Number. }\end{array}$ & Part Type & LDC & $\begin{array}{c}\text { Test } \\
\text { Level } \\
\text { (krads) }\end{array}$ & $\begin{array}{c}\text { Effective } \\
\text { Dose Rate } \\
(\mathrm{rads}) / \mathrm{s}\end{array}$ & $\begin{array}{l}\text { Param. } \\
\text { Degrad. } \\
\text { Level } \\
\text { (krads) }\end{array}$ & $\begin{array}{c}\text { Radiation Sensitive } \\
\text { Parameters }\end{array}$ & Report Number & Comments \\
\hline Maxim DG412 & $\begin{array}{l}\text { Analog } \\
\text { Switch }\end{array}$ & 9829 & 15 & 0.006 & $<2.5$ & $\begin{array}{c}I_{S S}, I_{\text {CO }}, I_{L L}, I_{\text {IH, }}, I_{\text {OOFF, }} \\
I_{\text {SOFF }}\end{array}$ & PPM-99-012 & Functional to $>15 \mathrm{krads}$ \\
\hline Maxim DG403 & $\begin{array}{l}\text { Analog } \\
\text { Switch }\end{array}$ & 9810 & $5-10$ & $\begin{array}{l}0.02 \\
0.003 \\
\end{array}$ & $<2.5$ & $\begin{array}{c}I_{D O}, I_{S S_{1}} I_{I H,} I_{t L} \text { and } \\
R_{O N}\end{array}$ & PPM-99-007 & $\begin{array}{l}\text { Failed functionality at } 7.5-20 \\
\text { krads }\end{array}$ \\
\hline Harris HI300 & $\begin{array}{l}\text { Analog } \\
\text { Switch }\end{array}$ & 9816 & $5-50$ & 0.03 & $<2.5$ & \begin{tabular}{|l}
$\mathrm{l}_{\mathrm{H}}, \mathrm{I}_{\mathrm{L}}$, and $($ for $>30$ \\
$\mathrm{krad}) \mathrm{I}_{\mathrm{SS}}, \mathrm{l}_{\mathrm{O}}$ and $\mathrm{R}_{\mathrm{ON}}$
\end{tabular} & PPM-99-005 & $\begin{array}{l}\text { Parts annealed at } 5.0 \text { and } \\
50 \mathrm{krad} \text {; functional despite } \\
\text { parametric degradation }\end{array}$ \\
\hline Harris HI506 & Multiplexer & 9745 & 50 & 0.04 & $>50$ & $\mathrm{~N} / \mathrm{A}$ & PPM-98-028 & \\
\hline
\end{tabular}

Table 6g: Summary of TID Test Results - Power Devices

\begin{tabular}{|l|l|c|c|c|c|c|c|c|}
\hline $\begin{array}{l}\text { Manufacturer \& } \\
\text { Part Number. }\end{array}$ & Part Type & LDC & $\begin{array}{c}\text { Test } \\
\text { Level } \\
\text { (krads) }\end{array}$ & $\begin{array}{c}\text { Effective } \\
\text { Dose Rate } \\
\text { (rads)/s }\end{array}$ & $\begin{array}{c}\text { Param. } \\
\text { Degrad. } \\
\text { Level } \\
\text { (krads) }\end{array}$ & $\begin{array}{c}\text { Radiation Sensitive } \\
\text { Parameters }\end{array}$ & Report Number & Comments \\
\hline $\begin{array}{l}\text { Lambda/ } \\
\text { Advanced Analog } \\
\text { ATR2815TF }\end{array}$ & $\begin{array}{l}\text { DC-DC } \\
\text { Converter }\end{array}$ & 9907 & $5-25$ & 0.022 & $>10$ & Efficiency, line reg & PPM-99-020 & \\
\hline $\begin{array}{l}\text { Interpoint } \\
\text { MTR2815 }\end{array}$ & $\begin{array}{l}\text { DC-DC } \\
\text { Converter }\end{array}$ & 9830 & $2.5-100$ & 0.09 & $15-50$ & Input current & PPM-98-031 & \\
\hline $\begin{array}{l}\text { Interpoint } \\
\text { MTR2805 }\end{array}$ & $\begin{array}{l}\text { DC-DC } \\
\text { Converter }\end{array}$ & 9828 & $2.5-100$ & 0.09 & $>50$ & Multiple parameters & PPM-98-030 & \\
\hline Linfinity SG1846 & $\begin{array}{l}\text { Pulse-Width } \\
\text { Modulator }\end{array}$ & 9715 & $20-200$ & 0.33 & $>80$ & $\begin{array}{c}\text { Error-amp section } \\
\text { parameters }\end{array}$ & PPM-98-C22 & \\
\hline
\end{tabular}

Table 6h: Summary of TID Test Results - Miscellaneous

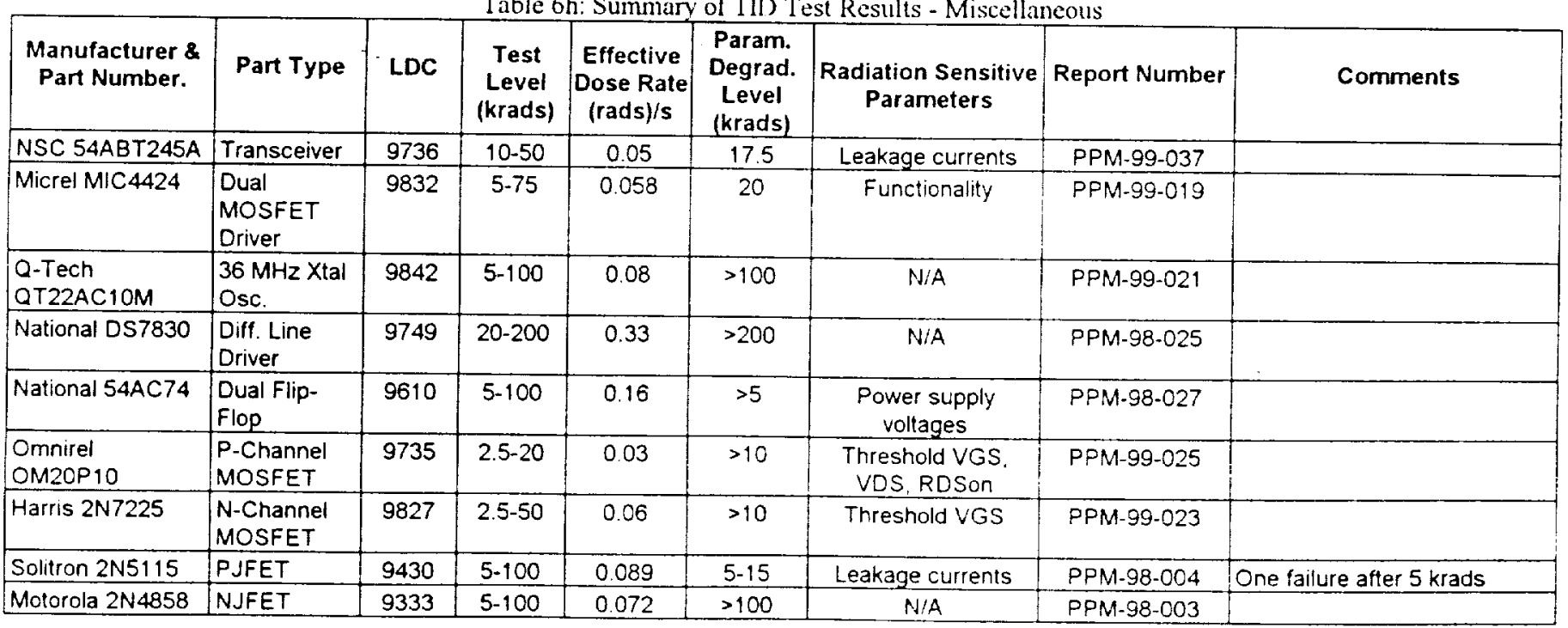

IEEE NSRECO0 Data Workshop - July 27, 200() - Reno. Nayada 


\section{SEE TEST REsUlts AND DISCUSSION}

\section{Power Devices}

1) TL7702B, TLC7705, and TL7705B Heavy Ion Testing

Texas Instruments power supply supervisors TL7702B. TLC7705, and TL7705B were tested for SEE at BNL. Figure 1 gives the measured (uncorrected) high-to-low SET cross section versus the linear energy transfer (LET) for TCL7705. Heavy ion-induced SET measured cross section data for TL7705B is shown in Figure 2, and data for TL7702B is shown in Figure 3. These tests were application specific. Details of the test techniques can be found in the test synopsis [2]. We visual observed that the SETs were typically $25 \mathrm{~ms}$ in duration and the dropout was all the way to ground. Considering corrections for application specific test factors, the value of the cross section measured with the automated collection system could be low by an order of magnitude. No low to high SETs were observed.

The TLC7705 did not experience any SELs for the test setup used.

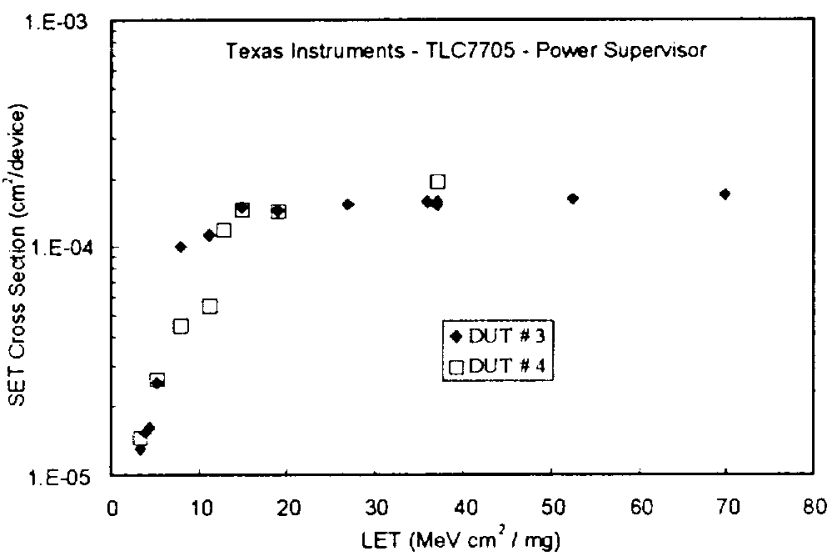

Fig. 1. Heavy ion-induced SET measured cross section data for two devices. The actual cross section is unknown and could be as high as an order of more large than the measured cross section.

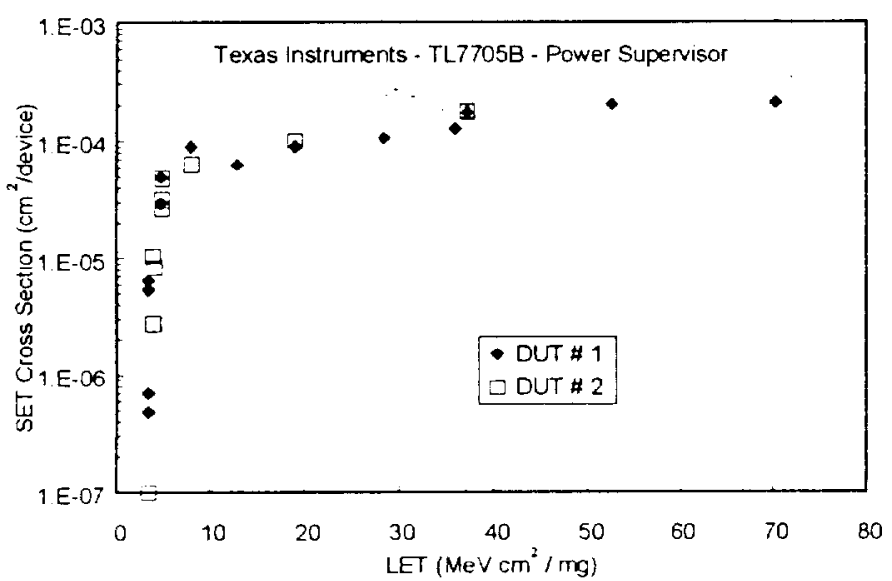

Fig. 2. Heavy ion-induced SET measured cross section data for two devices. The actual cross section is unknown and could be as high as an order of more large than the measured cross section

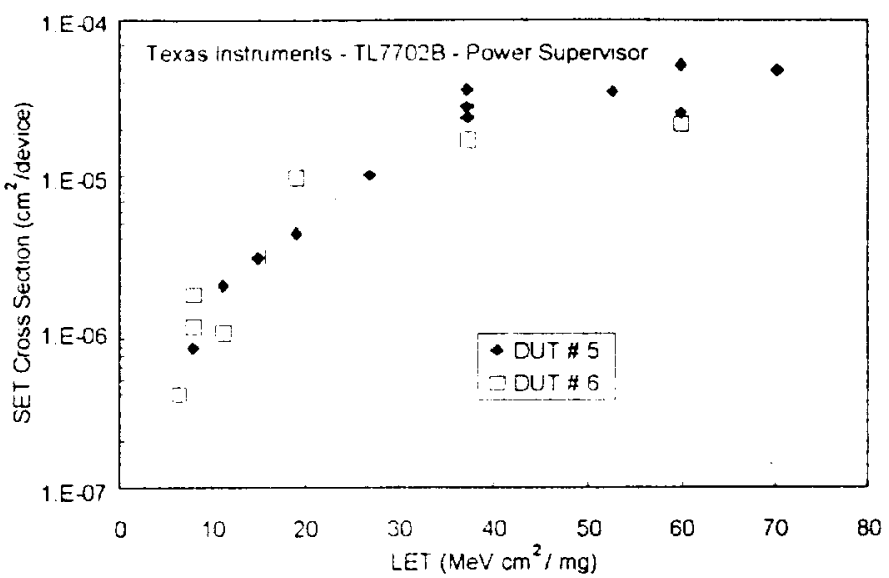

Fig. 3. Heavy ion-induced SET measured cross section data for two devices. The actual cross section is unknown and could be as high as an order of more large than the measured cross section.

\section{2) TL7770-5 Heavv Ion Testing}

Radiation-induced SET and SEL susceptibility of the Texas Instruments TL7770-5 Dual Power-Supply Supervisors was performed at BNL Single Event Upset Facility. Output of the device was monitored for radiation induced errors. Only undervoltage conditions were tested. The power supply current was monitored for large current increase and the device functionality was monitored.

The TL7770-5 did not experience any SELs for the test conditions and circuit configuration used, see test synopsis [3] for details of testing conditions). High to low SETs were observed, however, they were too short in duration to be captured by the automated duta collection system. They were $<2 \mu$ in duration and the pulse height varied from just visible on the digital oscilloscope to going all the way to ground. No low to high SETs were observed.

\section{3) TL7702B and TL7705B Proton Testing}

Proton induced degradation testing on Texas Instruments power supervisors TL7702B and TL7705B was performed at IUCF.

No errors, anomalics, or destructive conditions were observed on 2 test silmples of the TL7702B at a cumulative fuence of $6.56 \times 10^{11}$ protons $/ \mathrm{cm}^{2}$ ( $40 \mathrm{krads}$ (Si) of TID). Thus, the limiting device cross-section is $1.52 \times 10^{-12}$ $\mathrm{cm}^{2} /$ device. No obvious degradation due to TID or displacement damage was observed.

No errors, anomalies, or destructive conditions were observed on 2 test simples of the TL7705B at a cumulative fluence of $6.56 \times 10^{11}$ protons $/ \mathrm{cm}^{2}$ ( $40 \mathrm{krads}$ (Si) of TID). Thus, the limiting device cross-section is $1.52 \times 10^{-12}$ $\mathrm{cm}^{2} /$ device. No obvious degradation due 10 TID or displacement damige was observed. 
13. II) 'Dile:

1) Maxim MA7225 \&-bit digital to analog comverter

Testing was performed at BNL to determine the radiationmduced latch-up sensitivity of the Maxim MX7225 and to me:isure sensitivity as a function of supply voltage.

The MX7225 did not experience any SELs for the application specific test conditions used. Details of the lest setup can be found in [H]. Thus, the DUT has an LET $_{\text {h }}>$ $84.7 \mathrm{MeV} \cdot \mathrm{cm}^{2} / \mathrm{mg}$ with a limiting cross-section $<1 \times 10^{-4} \mathrm{~cm}^{2}$. No SETs were observed.

3) Analog Devices AD571S analog to digital converter

The device was monitored for latchup induced high power supply currents by exposing it to a number of heavy ion beams at BNL. Supply current was monitored for an increase or decease. No SELs were observed for the AD571S analog to digital converter up to LET of $60 \mathrm{MeV} \cdot \mathrm{cm}^{2} / \mathrm{mg}$. 3) Linear Technologies LTCI 419 analog to digital
converter

The Linear Technologies LTC1419 was monitored for latchup induced high power supply currents by exposing it to a number of heavy ion beams at BNL [5]. Supply current was monitored for an increase or decease.

No SELs were observed on 3 devices of each type tested under bias conditions of 7 volts at all ion LETs to a fluence of $1 \times 10^{7} \mathrm{p} / \mathrm{cm}^{2}$.

1) Analog Devices DACO8 digital to analog converter

The Analog Devices (AD) DAC08 digital to analog converter was tested at BNL to determine the single event transient and latch-up sensitivity. Tests were perforned to screen for the possibility of upset and latch-up and measure sensitivity as a function of input code (output voltage) and particle LET. The DAC was operated in a dc mode as required by the application specific test (dc output mode only).

The devices were exposed to a fluence of $10^{7}$ particles $/ \mathrm{cm}^{2}$ of Titanium, Bromine and Iodine ions with no single event upsets or latchup. An occasional trigger on the digital scope was seen, indicating a possible upset. Upon review of the data it was determined that these events were just noise. The upset window was kept intentionally small to catch even upsetting the LSB so temporary fluctuations of noise could trigger the scope. The events seen were very infrequent (approximatcly 15 events in $2 \times 10^{8}$ ions) and not considered to have a detrimental impact to the testing. The Analog Devices (AD) DAC08 Digital to Analog Converter is considered to have an LET $_{\text {th }}$ for upsets and latchup greater than 119.6 $\mathrm{MeV} \cdot \mathrm{cm}^{2} / \mathrm{mg}$. This latchup result is valid for generic usc, but since this testing was dc output mode only, the upset portion of this testing is application specific to operation in the de output mode [6].

\section{$\therefore$ Fairchild R20793 Programmable Read Only Memory (PROMI)}

Test conditions included the nominal case level for the Supply Voltage ( $\mathrm{VS}_{\mathrm{S}}=5$ Volts) and a clieckerboard pattern (55AA) while the device operalcd at a frequency of $1 \mathrm{MHz}$.

The devices were exposed to a fluence of $5 \times 10^{5}$ to $1 \times 10^{6}$ particles $/ \mathrm{cm}^{2}$ of Brominc and various fluences of the Carbon, Silicon, and Chlorine ions with no single event latchups. The Fairchild R29793 PROM is considered to have an $\mathrm{LET}_{\text {th }}$ for latchup greater than $37 .+\mathrm{MeV} \cdot \mathrm{cm}^{2} / \mathrm{mg}$.

Upsets, on the other hand, were common. During the testing, it became apparent that there were actually two modes of upsets. These were single cell upsets and massivedevice-failure upsets (where approximately all storage locations would read incorrectly). For the single cell upsets the approximate $\mathrm{LET}_{\mathrm{th}}$ is $3 \mathrm{MeV} \cdot \mathrm{cm}^{2} / \mathrm{mg}$ and the device saturation cross section is $3 \times 10^{-3} \mathrm{~cm}^{2}$. For the massive error events, approximate $\mathrm{LET}_{\text {th }}$ is $5 \mathrm{MeV} \cdot \mathrm{cm}^{2} / \mathrm{mg}$ and the device saturation cross section is $1 \times 10^{-4} \mathrm{~cm}^{2}$.

For the massive error events, the ion beam flux was allowed to continue until the error counter jumped, nearly instantaneously, to in excess of 70,000 errors. On occasion, before the beain could be stopped, a second massive event was observed as the counter jumped to in excess of 140,000 . From Figure 4, it can be secn that for both normal and angled incident Carbon ion bcams, no massive upset events were observed. At the higher LETs of 7.88 and 11.4, all the devices experienced these crrors at a highly variable rate [7].

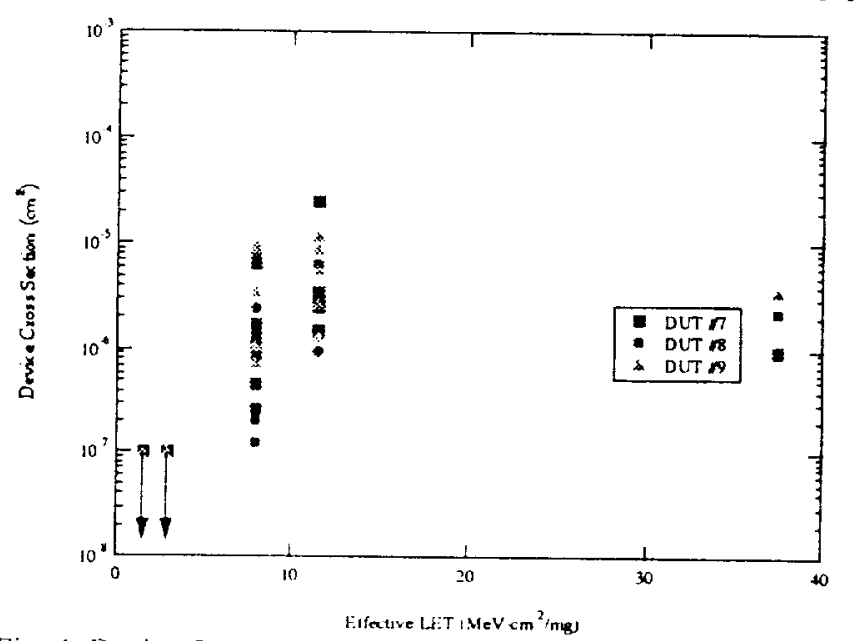

Fig. 4. Device Cross Section as a function of Effective LET for the massive error events. Data points with a down-pointing arrow indicate that no events were observed under those conditions.

D. Digital Signal Proceswors

1) Lockheed-Martin R//20120

Lockhoed-Martin RH21020s were tested for SEU under irradiation by proton beams in both dynamic and static modes [8]. Outpuls from the DUT were compared to those from an identical, non-irradiated reference chip and logged as errors when they did not match. The DUTs were operated at $15 \mathrm{MH} /(50 \%$ derated) and a power supply voltage of 5 volts. They were irradiated with proton nuences up to $1.4 \times 10^{12}$ 
particles per $\mathrm{cm}^{2}$ at fluxes ranging from $8.6 \times 10^{7}-1.7 \times 10^{3}$ particles $/ \mathrm{cm}^{2}$ per second. The total upset cross section of the DSP for protons was measured to be $7.64 \times 10^{-11} \mathrm{~cm}^{2}$ per device. The total device cross section broke down is $5.6+\times 10^{-11} \mathrm{~cm}^{2}$ per device for the Data Address Gencrator registers and $2.29 \times 10^{-11} \mathrm{~cm}^{2}$ for the General Purpose Registers. No upsets were observed for the Multiplier Registers, System Registers. Internupt Registers or $\mathrm{I} / \mathrm{O}$ portions of the device, implying a proton upset cross section less than $7.14 \times 10^{-13} \mathrm{~cm}^{2}$ per device for these portions of the chip. Table 7 below summarizes the SEE test results for both the Lockheed-Martin RH21020 and the Temic Semiconductor TSC21020F. No latchup events were observed, consistent with the results of heavy-ion upset and latchup test results reported previously [9].

2) Temic Semiconductor TSC21020F

Temic Semiconductor TSC21020Fs were tested for SEU under irradiation by proton beams in both dynamic and static modes [8]. They were irradiated with proton fluences up to $1.4 \times 10^{12}$ particles per $\mathrm{cm}^{2}$ at fluxes ranging from $8.6 \times 10^{7}-$ $1.7 \times 10^{9}$ particles $/ \mathrm{cm}^{2}$ per second. The total upset cross section of the DSP for protons was measured to be $9.9 \times 10^{-13}$ $\mathrm{cm}^{2}$ per device. The cross section for the $\mathrm{I} / \mathrm{O}$ is $9.9 \times 10^{-13} \mathrm{~cm}^{2}$ per device. No upsets were observed for the Data Address Generator registers, Multiplier Registers, System Registers, or Interrupt Registers portions of the device, implying a proton upset cross section less than $9.9 \times 10^{-13} \mathrm{~cm}^{2}$ per device for these portions of the chip.

It was noted during irradiation that some anomalous current signatures on the TSC21020F DSP occurred. No functionality of the DUT appeared to be impaired. The two samples of the TSC21020F that were exposed to the proton beam began showing increases in power supply lcakage current at $\sim 100 \mathrm{krads}$ (Si) with excessive levels (out of specification) appearing at $>125 \mathrm{krads}$ (Si).

Table 7 below summarizes the SEE test results for both the Lockheed-Martin RH21020 and the Temic Semiconductor TSC21020F. All errors observed on both device types were data errors only. No errors requiring reset pulses were observed to maximum test fluences.
Table 7: Proton SFil: R1121020 and TSC21020F Area Test Results

\begin{tabular}{|c|c|c|}
\hline Type of Error & $\begin{array}{c}\text { Cross-section in } \\
\mathbf{c m}^{2} / \text { device } \\
\text { LMFS RH21020 }\end{array}$ & $\begin{array}{c}\text { Cross-section in } \\
\mathbf{c m}^{2} / \text { device } \\
\text { Temic TSC21020F }\end{array}$ \\
\hline Overall DUT & $793 \times 10^{-11}$ & $9.90 \times 10^{-13}$ \\
\hline $\begin{array}{c}\text { Data Address } \\
\text { Generator }\end{array}$ & \\
\hline (DAG) Registers & $5.64 \times 10^{-11}$ & $4.95 \times 10^{-13}$ \\
\hline $\begin{array}{c}\text { General Purpose } \\
\text { Registers (GPS) }\end{array}$ & $2.29 \times 10^{-11}$ & $4.95 \times 10^{-13}$ \\
\hline Multiplier Registers & $7.14 \times 10^{-13}$ & $4.95 \times 10^{-13 *}$ \\
\hline System Registers & $7.14 \times 10^{-13}$ & $4.95 \times 10^{-13}$ \\
\hline Interrupt Registers & $7.14 \times 10^{-13 *}$ & $4.95 \times 10^{-13 *}$ \\
\hline I/O errors & $7.14 \times 10^{-13}$ & $9.90 \times 10^{-13}$ \\
\hline
\end{tabular}

* indicates limiting cross-sections measured: no upsets observed in these areas of the device to the maximum test fluence

\section{E. Logic Devices}

\section{1) Motorola 10502 ECL Multiple NOR Gate}

The Motorola 10502 ECL Multiple NOR Gate was screened for the possitility of upset and latch-up and measure sensitivity as a function of particle LET. Test conditions included the nominal and worst case levels for the supply voltage (Vs $=-5.2$ and -7 Volts). One input to the NOR gate was maintained at ground potential while the other was clocked at $100 \mathrm{kHz}$ (as required by the HST application). A fluence of at least $1 \times 10^{7}$ ions $/ \mathrm{cm}^{2}$ was used at each test condition. The beam flux range of $2.5 \times 10^{4}$ to $1.1 \times 10^{5}$ particles $/ \mathrm{cm}^{2} / \mathrm{s}$ resulted in individual exposures of about between 1.6 and 6.7 minutes.

The input voltage conditions were evaluated at 7 different values of Linear Energy Transfor (LET). Testing began with a normal incident LET of $59.8 \mathrm{MeV} \cdot \mathrm{cm}^{2} / \mathrm{mg}$ obtained with Iodine ions. Following this, the Bromine beam provided a normal incident LET of $37.3 \mathrm{MeV} \cdot \mathrm{cm}^{2} / \mathrm{mg}$. Finally, the Nickel bciln provided a normal incident LET of 26.6 $\mathrm{MeV} \cdot \mathrm{cm}^{2} / \mathrm{mg}$. Angle of incidence was used to obtain the seven effective LETs used. Four samples from the same lot and date code were tested under overlapping sets of conditions.

From Figure 5, it can be scen that for most LET conditions. the cross section data is fairly constant, but this is likely due to the statistical variations. At the lower LET values between 20 and 40, there are cases where no events were observed. For all cases with effective LET greater than t), upsets were seen. 


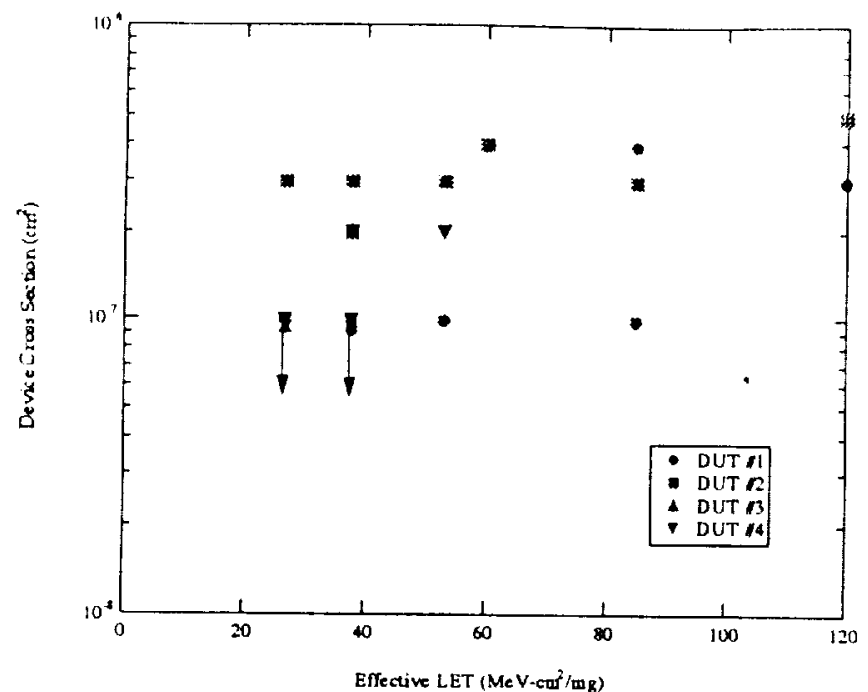

Fig. 5. Device Cross Section as a function of Effective LET. Data points with a down-pointing arrow indicate that no events were observed under those conditions.

The Motorola ECL Multiple NOR Gate is considered to have an $\mathrm{LET}_{\text {th }}$ for latchup greater than $119.6 \mathrm{MeV} \cdot \mathrm{cm}^{2} / \mathrm{mg}$ and the approximate $\mathrm{LET}_{\mathrm{th}}$ for upset is $20 \mathrm{MeV} \cdot \mathrm{cm}^{2} / \mathrm{mg}$ with a device saturation cross section is $5 \times 10^{-7} \mathrm{~cm}^{2}$. This latclup result is valid for generic use, but since this testing was done at a slow clock speed $(100 \mathrm{kHz})$ and one grounded input, the upset portion of this testing is application specific to this operational mode only [10].

\section{2) Motorola MC74LCX08 2INP and Gate}

The device was monitored for latchup induced high power supply currents by exposing it to a number of heavy ion beams at BNL. Supply current was monitored for an increase or decease. No SELs were observed for the MC7+LCX08 up to LET of $60 \mathrm{MeV} \cdot \mathrm{cm}^{2} / \mathrm{mg}$.

\section{F. Fiber Optic Links}

\section{1) HP HFBR-53D5 Transceiver}

We conducted extensive proton single event effects testing of the optical fiber-based HP HFBR-53D5 Gigabit Ethernet Transceiver with $850 \mathrm{~nm}$ VCSEL, Si PIN detector and a Si bipolar transimpedance amplifier (TIA). Throughout all tests the proton energy incident on the package was $63 \mathrm{MeV}$ to assure penetration and knowledge of the proton energy at the circuit location. Test variables included proton angle of incidence, optical power incident on the receiver, data rate up to $1100 \mathrm{Mbps}$, and part to part variation. Results indicated large cross-sections for the receiver with characteristics consistent with transients in the photodiode. The crosssection tended approximately linearly with data rate and was also reduced at higher optical powers up to approximately IOX for a $10 \mathrm{~dB}$ increase in received power. Significant increases were noted in the cross section at grazing angle of incidence to the receiver photodiode. Under some test conditions, this exceeded two orders of magnitude as compared to normal incidence. Negligible change was noted after an integrated $63 \mathrm{McV}$ proton thence of over $3.8 \times 10^{12}$ cilin'.

The lest configuration had wo transceivers on a card with an optical fiber cable connecting them and ECL in/out connectors to the BCP bit error rate tester (BERT). The bit error cross sections were characterized using a pscudorandom sequence as a function of optical power and data rate at various incident angles. and the radiation susceptibility of both the transmitter and receiver circuitry was monitored.

No catastrophic failures were observed to an exposure level of $\sim 25$ krads (Si) at $63 \mathrm{MeV}$. Figure 6 illustrates representative data captured during these experiments

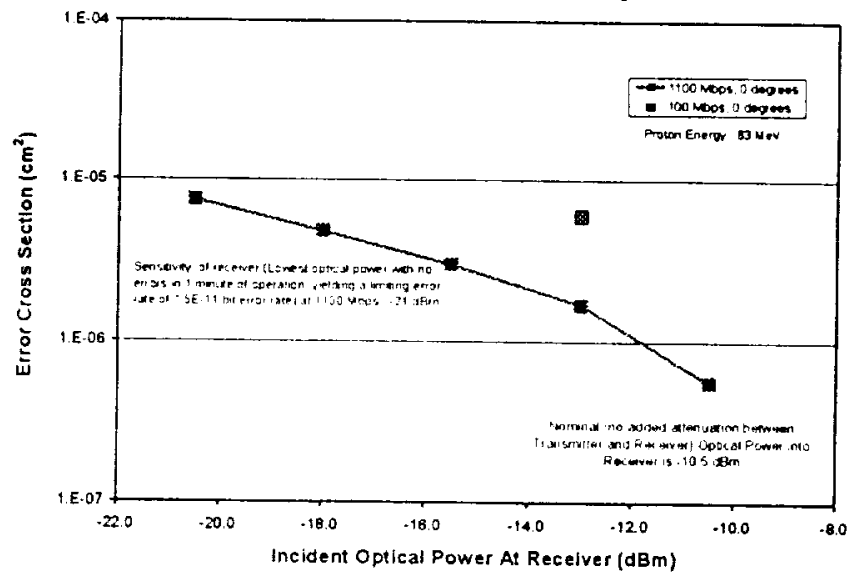

Fig. 6. shows the effect of received optical power and data rate on radiation-induced error cross section for the HFBR-53D5 device.

\section{2) Lasermate TTC-15.5M2 \& TTC-155M4}

We conducted extensive proton single event effects testing of the optical fiber-based $155 \mathrm{Mbps}$ transceivers TTC-155M4 (850 $\mathrm{nm}$ VCSEL transmit/1300 $\mathrm{nm}$ receive) and TTC-155M2 $(850 \mathrm{~nm}$ VCSEL transmit/850 nm Si PIN receive) fiber optic link hardware. Throughout all tests the proton energy incident on the package was $63 \mathrm{MeV}$ to assure penetration and knowledge of the proton energy at the circuit location. Test variables included proton angle of incidence, optical power incident on the receiver, data rate up to $1100 \mathrm{Mbps}$, and part to part variation. Results here also indicated large cross-sections for the receiver with characteristics consistent with transients in the photodiode. The cross-section tended approximately limearly with data rate and was also reduced at higher oplical powers up 10 approximately $20 \mathrm{x}$ for a $17 \mathrm{~dB}$ increase in received power. Significant increases were noted in the cross section at graking angle of incidence to the receiver photodiode. Under some test conditions, this exceeded two orders of mignitude as compared to normal incidence. Negligible change was noted after an integrated 63 $\mathrm{MeV}$ proton fluence of over $4.8 \times 10^{11} \mathrm{~cm}^{-2}$.

The test configuration had two transceivers on a card with an optical fiber cable connecting them and ECL invout connectors to the BCP bit error rate tester (BERT). The bit crror cross sections were characterized using a pseudorandom sequence as a function of optical power and data rate 
at various incident angles, and the radiation susceptibility of both the transmitter and receiver circuitry was monitored.

No catastrophic fililures were observed to an exposure level of $\sim 25$ krads (Si) at $63 \mathrm{McV}$ protons. Figure 7 illustrates representative data captured during these cxperiments. Cross Section vs Angle of Incidence

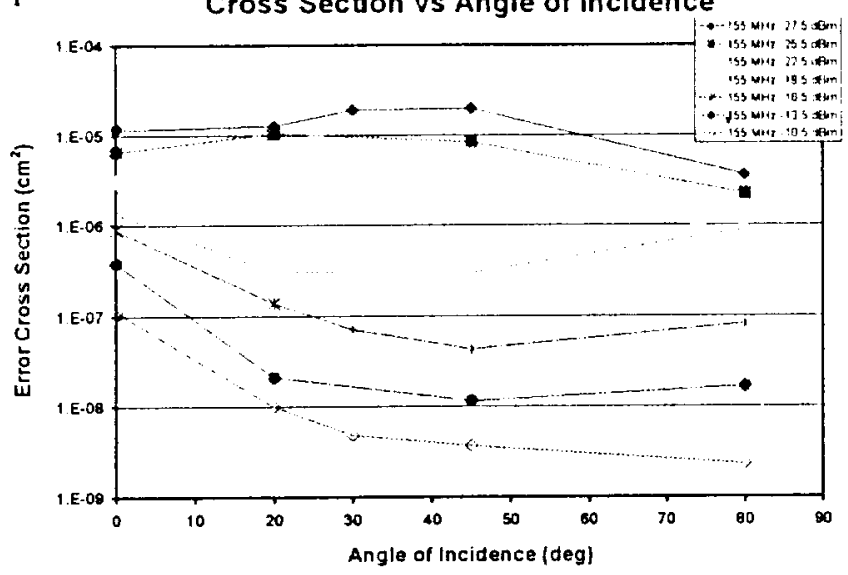

Fig. 7. Shows the measured effect of angular incidence and optical power on error cross section for the Lasennate TTC-155M4.

\section{G. Linear Bipolar Devices \\ 1) National Semiconductor CLC449Ultra-Hidehand Monolithic Operational Amplifier}

Tests were performed to screen for the possibility of latchup and measure sensitivity as a function of supply voltage and particle LET. Test conditions included a supply voltage (Vs) levels of $\pm 5 \mathrm{~V}$ and $\pm 5.5 \mathrm{~V}$. Supply currents were automatically monitored. The normally incident fluence was at least $9.5 \times 10^{6}$ ions $/ \mathrm{cm}^{2}$. The beam flux ranged from $1.2 \times 10^{4}$ to $1.0 \times 10^{5} \mathrm{particles} / \mathrm{cm}^{2} / \mathrm{s}$ resulted in individual exposures between 95 and 790 seconds. The DUT was loaded with 100 ohm resister. For all cases, the input was 2 Vpp and the output was $4 \mathrm{Vpp}$. The typical input frequency was 200 $\mathrm{MHz}$, the test setup limited the input frequency to $500 \mathrm{MHz}$.

Application specific SELs were observed. The CLC449 did not experience any SELs up to LET of $60 \mathrm{MeV} \cdot \mathrm{cm}^{2} / \mathrm{mg}$.

2) APEY PAO7 High Power OP AMP

The APEX PA07 was monitored for latchup induced high power supply currents by exposing it to a number of heary ion beams at BNL. Supply current was monitored for an increase or decease. No SELs were observed up to LET of 60 $\mathrm{MeV} \cdot \mathrm{cm}^{2} / \mathrm{mg}$.

\section{3) National Semiconductor LUC6081 Precision OP A.LP}

The National Semiconductor LMC6081 was monitored for latchup induced high power supply currents by exposing it to a number of heavy ion beams at BNL. Supply current was monitored for an increase or decease. No SELs were obsened up to LET of $60 \mathrm{MeV} \cdot \mathrm{cm}^{2} / \mathrm{mg}$.

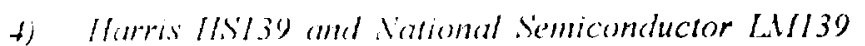
(immpersatom

A sudty has been undertaken using limear comparators from two vendors (Harris, now Intersil, and National Semiconductor) to collect a sufficient amount of data under many operational conditions in an attempt to understand the SET generation and characteristics. This information is to be utilized in the deselopment of this test methodology for comparators and possibly other linear devices.

Bolh LM-139 and HS-139 comparators produce SETs on lheir outputs. The cross sections and threshold LETs for the comparators are only slightly scnsitive to the applied bias. However, the LM-139 cross section and threshold LET has a strong dependence on the input differential voltage, first reported in I |. The HS-139 has an LET threshold of approximatcly $8-10$ and a cross section of $3 \times 10-4 \mathrm{~cm} 2$. The LM-139 has an LET threshold and cross section that vary with the input voltage differential from $1-10$ (LET) and I $x$ $10-5$ to $3 \times 10-4 \mathrm{~cm} 2$ (cross section). It should also be noted that the output transient characteristics (peak height and pulse width) of the LM-139 are also a strong function of input differential vollage.

5) Arational Semicombuctor Lull39 Comparator (Application Specific testing)

LM139 devices were exposed to a fluence of $1 \times 10^{6}$ to $1 \times 10^{7}$ particles $/ \mathrm{cm}^{2}$ of the Chlorine, Titanium, Nickel, Bromine and lodine ions with no single event latchups. The National Semiconductor LM139 is considered to have an LET threshold for latchup greater than $59.8 \mathrm{MeV} \cdot \mathrm{cm}^{2} / \mathrm{mg}$. For single event transients, the approximate LET threshold for high output is $20 \mathrm{MeV} \cdot \mathrm{cm}^{2} / \mathrm{mg}$ and the device saturation cross section is $2 \times 10^{-4} \mathrm{~cm}^{2}$. The approximate LET threshold of $20 \mathrm{MeV} \cdot \mathrm{cm}^{2} / \mathrm{mg}$ and device saturation cross section of $1 \times 10^{-4} \mathrm{~cm}^{2}$ is seen for the low output conditions. It must be noted that these results are application specific [11]. Test conditions were: $V_{c c}= \pm 7$ Volts and maintaining a two volt differential between the input voltages, $V_{+}=3$ Volts, V- was set to 1 Volt, or vice versa.

\section{6) Analog Devices .1.41962 Comparator}

The MAX962 was monitored for latchup-induced, high power-supply currents by exposing it to a number of heavy ion beams at BNL. Supply current was monitored for an increase or decease. No SELs were obsened up to LET of 60 $\mathrm{MeV} \cdot \mathrm{cm}^{2} / \mathrm{mg}$.

7) Analog Dovices Ab793SO Sample and Hold -lmplifier

The AD78. did not experience any SELs up to LET $_{\text {th }}>90$ $\mathrm{McV} \cdot \mathrm{cm}^{2} / \mathrm{mlg}$. Exposures were performed to a fluence of $1 \times 10^{-} \mathrm{p} / \mathrm{cm}^{2}$ or grealer. Figure 8 gives the results of SET application specilic lesting results on the Analog Devices AD783. The datia is plotted as the SET cross section versus the lincar energy lramsler (LET). During ksting we observed that the SETs were Iypically $<2 \mu$ s in duration. The height was Iy pically somewhere between $0.4 \mathrm{~V}$ and $1 \mathrm{~V}$, however we did obsene some that were larger. maybe as large as 2-3V. 
This is a qualitative summary of the data observed during the exposures [12].

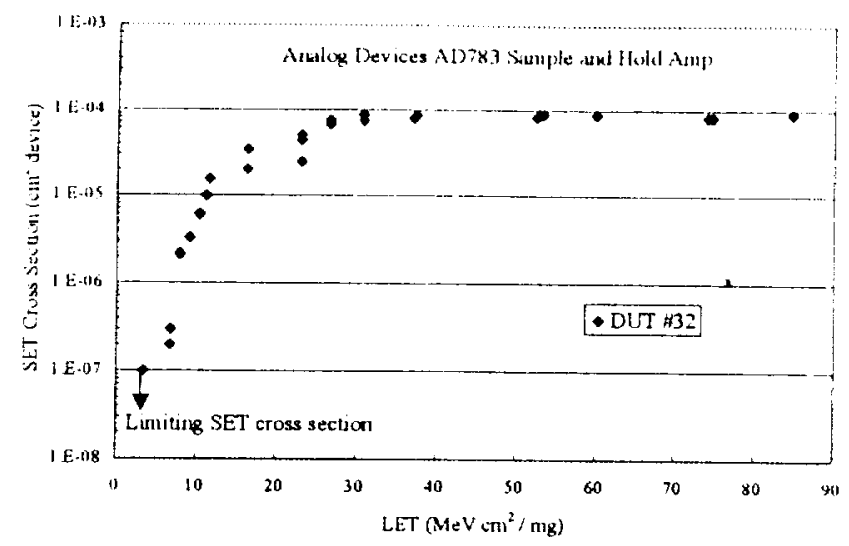

Fig. 8. Heavy ion SET cross section for AD783.

\section{8) Amptek A250 Charge Sensitive Amplifier}

The Amptek A250 was monitored for latchup induced high power supply currents by exposing it to a number of heavy ion beams at BNL. Supply current was monitored for an increase or decease. No SELs were observed up to LET of $60 \mathrm{MeV} \cdot \mathrm{cm}^{2} / \mathrm{mg}$.

\section{9) MSA-0670 MAIC Amplifier}

The Hewlett Packard MSA-0670 MMIC was tested for susceptibility to SET and SEL under irradiation with heavy ions $\left(265.9 \mathrm{MeV} \mathrm{Ni}\right.$ ions with $\mathrm{LET}=26.6 \mathrm{MeV} \cdot \mathrm{cm}^{2} / \mathrm{mg}$ and $343 \mathrm{MeV}$ I ions with $\mathrm{LET}=59 \mathrm{MeV} \cdot \mathrm{cm}^{2} / \mathrm{mg}$ ). The device was tested for both nominal (7.93 volts) and worst-case (8.03 volts) supply voltages, and with $V_{\text {in }}=0.5 \mathrm{~V}$ @ $150 \mathrm{MHz}$. No SET or SEL events were seen for ion fluences of at least $10^{7}$ particles $/ \mathrm{cm}^{2}$ at fluxes from $4.6 \times 10^{4}$ to $1.1 \times 10^{5}$ with effective LETs up to $84.6 \mathrm{MeV} \cdot \mathrm{cm}^{2} / \mathrm{mg}$ [13].

\section{H. Optocouplers}

\section{I) Isolink OLH560I}

Proton SET testing was performed at TRIUMF at 68,103 , 160. $225 \mathrm{MeV}$ and CNL $63 \mathrm{MeV}$. We observed SETs for various angles of incidence relative to the photodiode. The cross section increased with angle as it was swept around grazing angle for the photodiode for the 103,63 and the 68 $\mathrm{MeV}$ beam. The angular effect was not observed for the higher energies.

\section{2) Micropac 6N/3t}

Proton SET testing was performed at TRIUMF at 68.103 . 160, $225 \mathrm{MeV}$. We observed SETs for various angles of incidence with the cross section increasing the angle was near grazing angle for the photodiode at all energies.

\section{Others}

\section{1) Dallas Semiconductor DS1670E System Controller}

The DS1670E experience several SELs with several ions at several angles of incidence. The latchup current ranged from to to $109 \mathrm{~mA}$. During the latchup condition the desice was not functional, however the device recovered after il power cycle
Figure (1) shows the device cross section for an SEL at various LETs. The incident particle beam angle relative to the die wias chinged to oblain efrective LETs between those listed in the table. The $3.3 \mathrm{~V}$ cxposures are indicated by open circles, and the $3.6 \mathrm{~V}$ exposures are denoted by the solid triangles. The data crossed by a solid horizontal line indicate that no events where observed during the exposure, i.e. limiting cross section [1+].

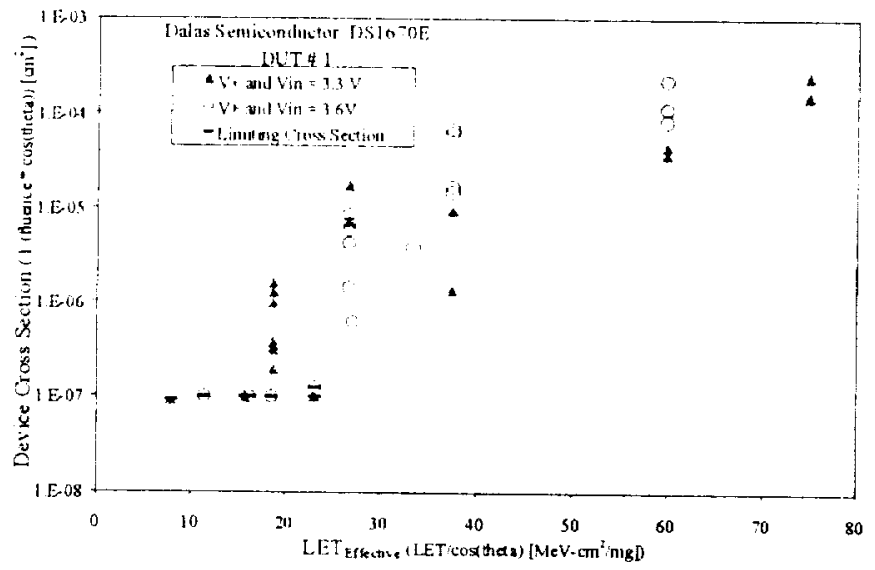

Fig. 10. Heavy Ion SEL cross section for DS1670E.

\section{2) TISN54LVTHI62+4A Buffer/Drivers}

Texas Instruments SN54LVTH16244A was monitored for latchup induced high power supply currents by exposing it to a number of heavy ion beams at BNL. Supply current was monitored for an increase or decease. No SELs were observed up to LET of $60 \mathrm{MeV} \cdot \mathrm{cm}^{2} / \mathrm{mg}$.

\section{3) National Siemiconductor CGS74LCT2524 Clock Driver}

The CGS74LCT252t was monitored for latchup-induced, high power-supply currents by exposing it to a number of heavy ion beams at BNL. Supply current was monitored for an increase or deceasc. No SELs were observed up to LET of $60 \mathrm{McV} \cdot \mathrm{cm}^{2} / \mathrm{mg}$.

\section{t) Micrel MIIC+423.MOSFET Driver}

Tests were performed to screen for the possibility of latchup and measure sensitivity as a function of input voltage. input frequency, case temperature, and particle LET. A normally incident fluence at leist $1 \times 10^{7}$ ions $/ \mathrm{cm}^{2}$ was used at each test condition. A beam nux of $8.2 \times 10^{5}$ to $1.4 \times 10^{5}$ particles $/ \mathrm{cm}^{2} / \mathrm{s}$ resulted in individual exposures between 75 and 130 seconds. A $100 \mathrm{kHz}$ signal was placed on the input oscillating between $-5 \mathrm{~V}$ and $+12 \mathrm{~V}$. DC input signals of $+12 \mathrm{~V}$ and $-5 \mathrm{~V}$ was also used. For all cases the supply voltage $\left(\mathrm{V}_{\mathrm{s}}\right)$ wals level of $12 \mathrm{~V}$. The MIC4+23 did not experience any SELs for the test conditions described and circuit configuration described above. Detailed test conditions for each exposure can be found it reference [15].

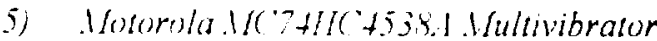

The Molorola MC $7+\mathrm{HC}+538 \mathrm{~A}$ was monitored for latchupindeced. high power-supply currents by exposing it to a number of heity ion beans an BNL. Supply current was 
monitored for an increase or decease. No SELs were observed up to LET of $60 \mathrm{MeV} \cdot \mathrm{cm}^{2} / \mathrm{mg}$.

\section{6) Dallas Semiconductor DS\$1803 Addressahle Dual Digital Potentiometer}

Tests were performed on the DS1803 to screen for susceptibility to latch-up and measure sensitivity as a function of supply voltage and particle LET. Test conditions included nominal and worst-case levels for the supply voltage $\left(V_{c c}\right)$ of $3.3 \mathrm{~V}$ and $5.5 \mathrm{~V}$. A normal incidence fluence of at least $1 \times 10^{7}$ ions $/ \mathrm{cm}^{2}$ was used at each test condition unless an SEL occurred. A beam flux range of $2 \times 10^{2}$ to $1.3 \times 10^{5}$ particles $/ \mathrm{cm}^{2} / \mathrm{s}$ resulted in individual exposures between 10 second and 13 minutes. Both input voltage conditions ( $3.3 \mathrm{~V}$ and $5.5 \mathrm{~V}$ ) were evaluated at 4 different ions and a several angles of incidence.

Device functionality was monitored by observing the resistance of the potentiometer. If the device current experienced a sudden increased larger than $I_{L}$, the power was cycled and the DUT was checked for functionality, we called this an SEL. The DUT functionality information was not saved to a file. From time to time during the exposure, but before an SEL, the device was monitored for changes in the output (this is known as a single event upset SEU). A description of our observation is provided below. This information is provided as a cursory look at the SEU susceptibility of the device. The number of SEUs were not captured. Therefore the rate of occurrence in a space flight application can not be predicted.

The DS1803 experienced several SELs with several ions at several angles of incidence. The latchup current ranged from 51 to $57 \mathrm{~mA}$. During the latchup condition the device was not functional, however the device recovered after a power cycle [16].

Figures 11 shows the device cross section for an SEL at various LETs. The $3.3 \mathrm{~V}$ exposures are indicated by open circles, and the $5.5 \mathrm{~V}$ exposures are denoted by the filled in triangles. The data crossed by a solid horizontal line indicate that no events where observed during the exposure, i.e. limiting cross section.

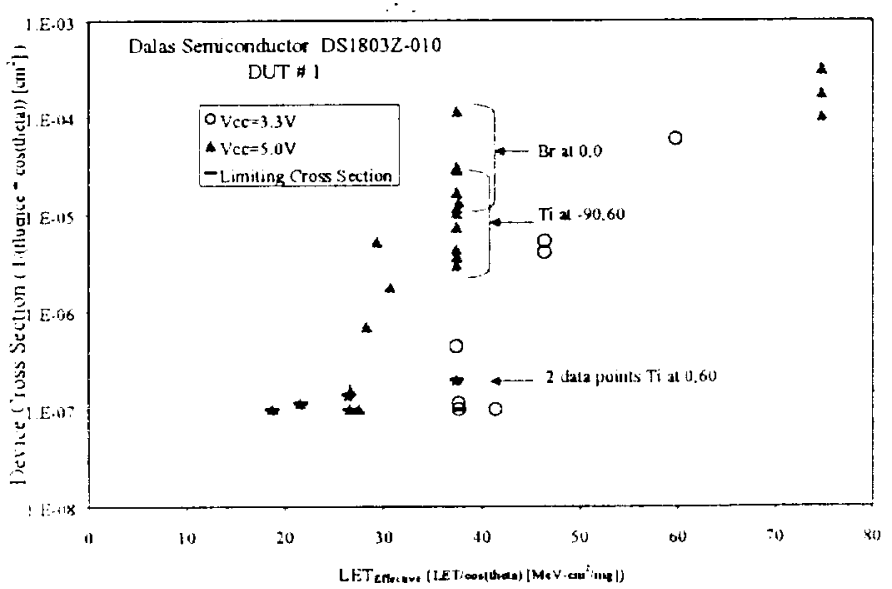

Fig. 11. DS180.3 cross section for an SEL at various LETS

\section{DISI'LACHMINAT DAMAGE TEST RESULTS AND Discussion}

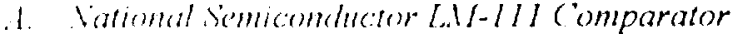

It has been demonstraled that some linear devices are susceptible to culanced degradation when exposed to proton suvironments as compared to Cobalt-60. In an effort to understand this effect and develop an efficient test procedure, National Semiconductor Corporation (NSC) LM-111 Comparators have been exposed to proton environments at Indiana University (IU) and University of California at Davis (UCD) cyclotrons. The initial parameter investigated and the most sensitive is the input current. For consistency, this current was measured at what is termed the crossover point (one input is held at a voltage while the other is swept from the negative to positive side of that voltage. The crossover current is the point where the two input currents are equal).

To develop a complete data set for this investigation, rail voltages of \pm 15 Volts, \pm 10 Volts, \pm 5 Volts and $+15 / 0$ Volts were used. For all of these bias conditions, a crossover current was measured on six devices at input voltages from 3 to +3 Volts at 1 Volt increments. Examples of the data set are shown in figures 12 and 13. As can be seen, significant bias dependence is observed for both the rail bias and the input conditions.

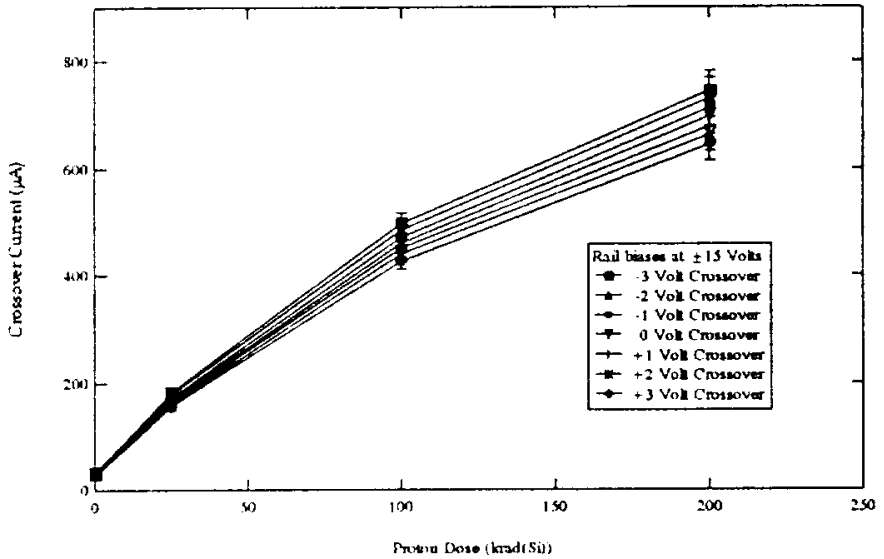

Figure 12. Crossover currents as a function of proton dose with a rail bias of \pm 15 Volts. The data points are the average response of six parts and the error bars represent one sigma variation.

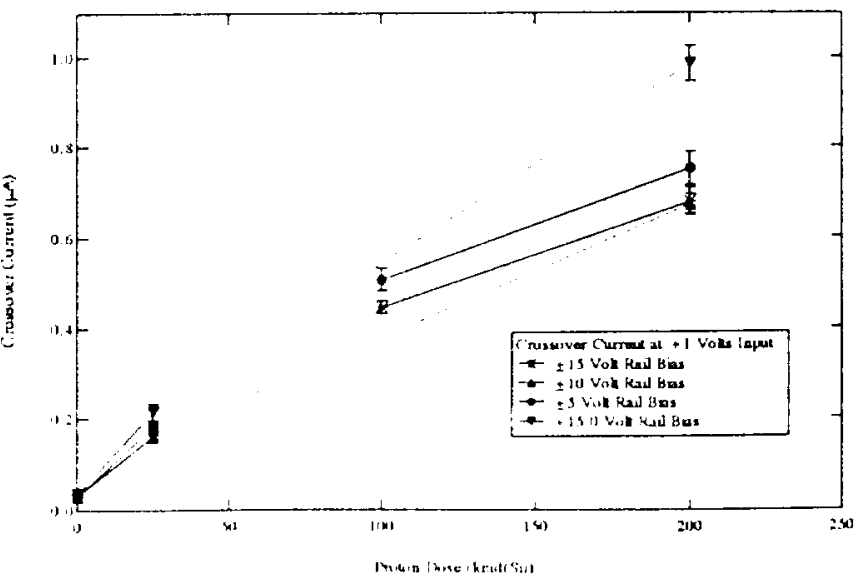


Figure 13. Crossover currents as a finction of proton dose with crosisover incisured at +1 Volts at the inputs. The datil points are the average response of six parts and the error bars represent one stgma vartation.

\section{Optocouplers \\ 1) Isolink OLIIL +9}

The OLH249 was irradiated with $195 \mathrm{MeV}$ protons at IUCF. IF was swept from 4 to $26 \mathrm{~mA}$ with $\mathrm{V}_{\mathrm{CE}}=5 \mathrm{~V}$. CTR degradation was observed at $6 \times 10^{11} \mathrm{p} / \mathrm{cm}^{2}$.

\section{2) Mficropac 66099}

Proton effects characterization of the Micropac 66099 optocoupler were made at Crocker Nuclear Laboratory at the University of California at Davis using $63 \mathrm{MeV}$ protons. Three devices (DUTs 1,2,3) were used for a quick set of measurements to ensure that reasonable choices were made for the proton fluences. A very detailed set of measurements were completed for an additional 3 devices (DUTS 4,5,6). The quick look was designed to be a worse case look and was performed for a no load condition with $\mathrm{I}_{\mathrm{f}}$ at $1 \mathrm{~mA}$ and $5 \mathrm{~mA}$. The detailed measurements performed on a second set of 3 devices included CTR measurements at various loads $(0,430$ $\Omega, 970 \Omega$, and $2.7 \Omega$ ) on the output for $I_{f}$ from 0.5 to $20 \mathrm{~mA}$ (in $0.5 \mathrm{~mA}$ increments) for each $V_{C E}$. $V_{C E}$ itself was varied from 0 to $10 \mathrm{~V}$ in $1 \mathrm{~V}$ increments. Corresponding transistor measurements were also made. It is important to note that of the 6 devices tested, one exhibited anomalous beliavior, and performed significantly worse under irradiation.

There was a range of initial CTRs observed and the spread in values was dependent on the operating conditions. Figure 9 shows a typical data set for the CTR as a function of proton fluence for various operating conditions.

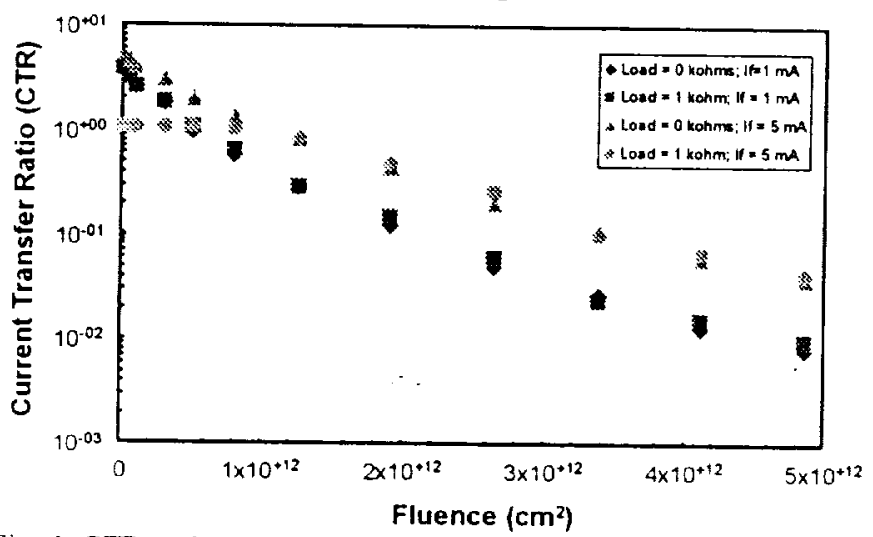

Fig. 9. CTR and transistor characteristics for the Micropatc 660199

\section{Optudiode ODS00 LED}

Proton-induced degradation testing of the Optodiode OD800 light emitting diode was performed. LEDs were cxposed to proton irradiations at University of California at Davis Crocker Nuclear Laboratory. The light output and $\mathrm{l}-\mathrm{V}$ characteristics of the device were monitored for radiationinduced degradation at various fluence levels. The Optodiode OD800 is a GaAs Double Heterojunction light emitting diode. Figure 14 shows the degradation $\left(\mathrm{P} / \mathrm{P}_{\mathrm{s}}\right)$ of the all the DUTs at each cxposure level (fluence) for $I_{f}=\operatorname{mA}$. The mcisured output power (P) for cach DUT at each fluence is normalized to the pre-irradiation output power $\left(P_{o}\right)$. Figure 15 shows the $[-V$ curves masured for DUT \#18. The solid dark line is the pre-irradiation values, the dash line is the post-irradiation values after an exposure $2.6 \times 10^{11} \mathrm{p} / \mathrm{cm}^{2}$. For the most part the post-and pre-rad values are the same. In the configuration used. the current resolution of the parametric analyzer is thonght to be $\sim \ln \mathrm{A}$. Measurements below $1 \mathrm{nA}$ should be considered to have large error bars. These data presented in Figure 2 are consistent with results on DUTs 1317. I-V curves were not measured for DUTs 1-12. See Reed et al., "Energy Dependence ... $\mid 17\}$.

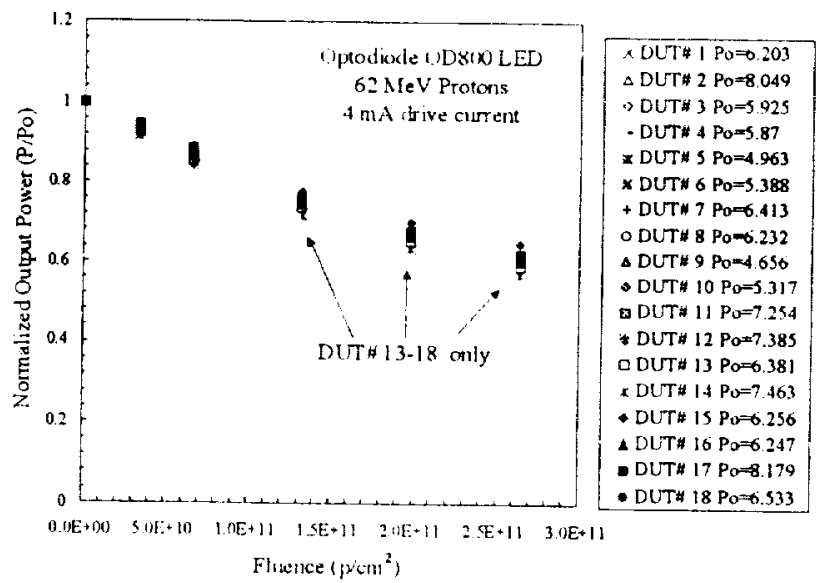

Fig. 14. Proton degradation of light output for 6 Optodiode OD800W. The output power was nomalized to the pre-rad values.

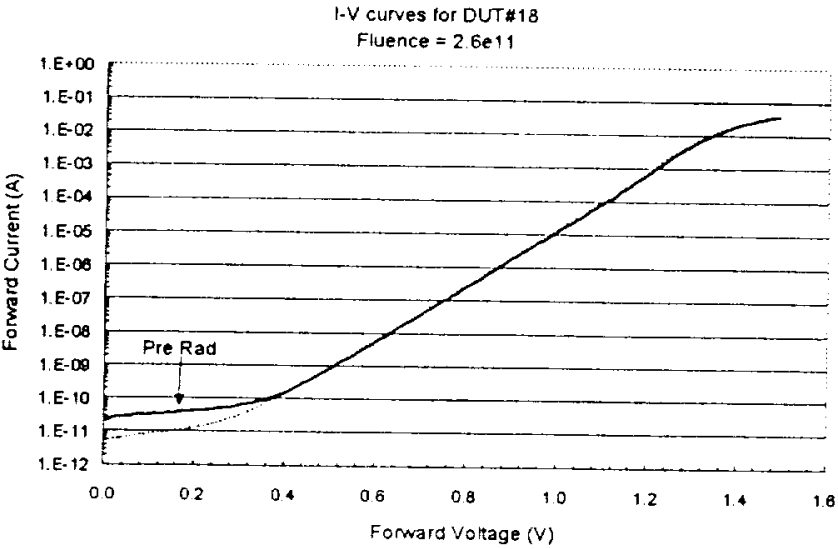

Fig. 15. Typical Optodiode OIJ800 DUTH17 post and pre-rad I-V curves.

\section{Ifonevell HFE-t0so a Vertical Cavity Surface Emitting Laser (l. SEL)}

The Honcywell HFE-408() ion-implanted 850 nm VCSELs were exposed unbiased to Cobalt-60 gamma irradiation to $\sim 18 \mathrm{Mrad}(\mathrm{Si})$ to ensure that the Ultem lens in the package would nol darken and obscure the proton test results. No significant gamma radiation-induced changes were obsened.

Proton tests were performed at TRIUMF in May 1999. and the Crocker Nuclear Laboratory (CNL) in June 1999. The VCSELs were irradiated unbiased which is a worse case 
since llice is no concurrent forward biascd annealing. As expected the primary eflect of proton exposure was an increase in the threshold drive current. For example, the initial threshold currents of $\sim 5 \mathrm{~mA}$ increased to $\sim 7 \mathrm{~mA}$ after a $63 \mathrm{MeV}$ exposure of $5 \mathrm{e} 13 \mathrm{~cm}^{\wedge}-2$. At the higher proton exposure levels, we also see a decrease in the slopes of the light output versus drive current curves (i.e. the dimerential quantum efficiency).

Additional $850 \mathrm{~nm}$ oxide-confined VCSELs with dillerent aperture sizes were also underwent proton characterization. The smallest threshold current shifts were obseried for the small aperture oxide-confined VCSELs. For example, the threshold current of the 4 micron squared oxide aperture $V C S E L$ remained almost unchanged at $-0.5 \mathrm{~mA}$ after a 63 MeV fluence of $5313 \mathrm{~cm}^{-2}$.

In summary, the VCSELs are very robust to gamma and proton irradiation and are suitable for most space applications.

\section{TID TEST RESULTS AND DISCUSSION}

\section{A. Comparators}

TID evaluations of three different comparators, Maxim's MAX913 and Analog Devices' (AD) CMP01 and PM139, revealed varying susceptibility. In addition to functionality; parametric measurements of quantities such as power supply current $\left(\mathrm{I}_{\mathrm{CC}}\right)$, input bias current $\left(\mathrm{I}_{\mathrm{b}}\right)$ offset voltage and current ( $V_{O S}$ and $\mathrm{I}_{O S}$ ), common-mode rejection ratio (CMRR), power supply rejection ratio (PSRR) and gain $\left(\mathrm{A}_{\mathrm{ol}}\right)$, were performed at each step during the irradiation and annealing processes.

\section{B. Actel AJ280A FPGA}

Actel A1280A CQ172B FPGAs (5962-9215601MYC) were irradiated at a rate of $0.01 \mathrm{rad}(\mathrm{Si}) / \mathrm{s}$ using a $\mathrm{Co}^{60}$ source. The parts were irradiated under bias to levels of 3.0, 5.0,10.0 and $15.0 \mathrm{krad}(\mathrm{Si})$. At levels above $5 \mathrm{krad}(\mathrm{Si})$, supply currents were seen to increase above specified levels. Substantial improvement in these parameters was seen after a 168 hour anneal at $25^{\circ} \mathrm{C}$.

\section{Operational Amplifiers.}

The Radiation Effects and Analysis Group undertook 27 TID evaluations on 23 different amplifiers, 12 from Analog Devices (AD), + from Linear Technologies (LT), 2 from National Semiconductor (NSC) and one each from Burr Brown. Apex, Amptek, Maxim and Omnirel. Failure levels ranged from less than 5 krads(Si) to over $200 \mathrm{krads}(\mathrm{Si})$. Functional and parametric tests were performed at every irradiation step.

\section{Analog-to-Digital and Digital-to-Analog Comverters}

The Radiation Effects and Analysis Group undertook evaluations on 4 ADCs and 4 DACs from Analog Devices and on onc DAC each from Maxim and Micronctworks. There was also one RMS-DC convener from Maxim. Parametric failure levels ranged from $>200 \mathrm{krads}(\mathrm{Si})$ to $<1$
krad(Si). For these devices, functional failures may be seen cen before significamt parametric degradation. At least one part (Analog Derices AD976) may cxhibit ELDRS.

\section{E. Voltuge References and lohtage Regulators}

Voltage references and regulators exhibit a wide range of susceptibilities to ridiation damage. The 4 voltage regulators and 2 vollage relerences lested by the Radiation Effects Branch are consistent with this obsenation.

\section{F. Memories}

The speeds and capacities of commercial memories make them attractive to designers. They exhibit a broad range of radiation tolerances.

\section{G. Analog Sivitches and Multiplexers}

Parts were irradiated in steps from $2.5 \mathrm{krad}$ to $5 \mathrm{krad}$. Prior to irradiation and after each step, tests were performed to measure supply currents, $I_{D D}$ and $I_{S S}$, input leakage currents with inputs high and low, $I_{\Pi H}$ and $I_{\mathbb{I L}}$, on-resistances, $R_{O N}$, and so on. The HI506 multiplexer showed no significant functional or parametric degradation for dose levels up to $50 \mathrm{krad}(\mathrm{Si})$, as well as after a 168 hour, $25^{\circ} \mathrm{C}$ anneal.

\section{H. Power Devices}

With the exception of the Linfinity PWM, the power devices tested were hybrid DC-DC converters, and they exhibit a range of radiation tolerances. The PWM exhibits good radiation tolerance.

1. Miscellaneous Devices

The devices in this category do not fit neatly into any of the other catcgories. They include discrete FETs, drivers, a crystal oscillator, a transceiver and a logic device. The failure levels are as diverse as the device types.

\section{SUMMARY}

We have presented recent data from SEE. Co-60 total ionizing dose (TID), and proton-induced damagefon mostly commercial devices. It is the authors' recommendation that this data be used with caution. We also highly recommend that lot testing be perfermed on any suspect or commercial device.

\section{ACKN( WLEDGEMLNTS}

The Authors would like to acknowledge the sponsors of this efrort: NASA Electronic Parts and Packaging Program (NEPP), Electronics Radiation Characterization (ERC) Project, and Office of the Chief Engineer.

\section{RIHERINCES}

11] NASNGSRC Radtation liflects and Analysis home page, http:/radlome gefichasian 
12| R. Reed, D. Ilawkins, J. Fonky, "Ileavy lon Sillgle Lvent Ellects Test Results for Three Texas Instruments Micropower Supply Voltage Supervisor," http://ridhome gsti.nasa.gov/ radhomed papers/ bl12599a pdf, October 1999.

[3] R. Reed, J. Foncy, D. Hawkins, "Heavy Ion Single Event Errects Test Results for the Texas Instruments TL7770-5 Dual Power-Supply Supervisors," http://Tadhome.gsfi. nilsa gov/ radhome/ papers/ bl 12599 b.pdf, October 1999.

[4] R. Reed, J. Fonney, D. Hawkins, "Heavy Ion Latch-up lest Results for the Maxim MX7225 8-Bit DAC," http:/radhome. gstic nasa. gov/ radhome/ papers/ b1 12499a.pdf, October 1999.

[5] Robert Reed, Paul Marshall, Curtis Dunsmore, Jim Founey and Hak Kim, "Single Event Latchup Testing of theLincir Technologies LTC1419 and L'TC1419A," http://radhome. gsfe. nasa. gov/ radhome/ papers/ b043099a.pdf, July 1999.

[6] Jim Howard, Robert Reed, Jim Forney, and Hak Kim, "Heavy Ion Upset and Latch-up Test Results for the Analog Devices DAC08," http://radhome. gsfc. nasa. gov/ radhome/ papers/ b082399a.pdf, July 1999.

[7] Jim Howard, Robert Reed, Hak Kin, and Jim Forney, "Heavy Ion Upset and Latch-up Test Results for the Fairchild R29793," http://radhome. gsfc. nasa. gov/ radhome/ papers/ b082599a.pdf, July 1999.

[8] Kenneth A. LaBel, Hak S. Kim, Paul W. Marshall, "Proton Single Event Effects Test Results for the LMFS RH2 1020 and Temic TSC21020F Digital Signal Processors (DSPs)," http://radhome. gsfc. nasa. gov/ radhome/ papers/ dl13099a.pdf, November 1999.

[9] M.V. O'Bryan K.A. LaBel, R.A. Reed, J.W. Howard, J. Barth, C. Seidleck, P. Marshall, C. Marshall, H.S. Kim, D.K. Hawkins, M. Carts, and K.E. Forslund, "Recent Radiation Damage and Single Event Effect Results for Microelectronics," NSREC'99 Data Workshop, pp. 1-14, July 1999.

[10] Jim Howard, Robert Reed, Jim Forney, and Hak Kim, "Heavy Ion Upset and Latch-up Test Results for the Motorola ECL Multiple NOR Gate," http://radhome. gsfe. nasa. gov/ radhome/ papers/ b082499a.puf, August 1999.

[11] Jim Howard, Robert Reed, Jim Foney, and Hak Kim, "Heavy Ion Transient and Latch-up Test Results for the National Semiconductor LM139," http://radhome. gsfe. nasa. gov/ radhome/ papers/ b082699a.pdf, August 1999.

[12] Robert Reed, JimForney, Donald Hawkins, "Heavy lon Sing]e Event Elfects Test Results for the Analog Devices AD78? Sample and Hold Amplifier," http://radhome, gstc. nasa. gov/ radhome/ papers/ bl 12499b.pdf, October 1999

[13] Jim Howard, Rober Reed, Jim Forney, and Hak Kim, "Ileavy Ion Transient and Latch-up Test Results for the HP MSA0670," http://radhome. gsfe. nasa. gov/ radhome/ papers/ bu 824996 bdf, October 1999

[14] Robert Reed, Pau] Marshall, Jim Forney, and Curtis Dunsmore, "Heavy Ion Latch-up Test Results for the Dallas Semiconductor Portable System Controller DS1670," http:/radhome. gste. nasa gov/ radhome/ papers/ b0430999a.pdt, June 1999

[15] Robut Reed, Hak Kin, Donald Iaukins, "lkivy Ion Latch-up Test Results for the Micrel MIC+423 MOSFET Jriver." http:/radlome gsti našal. gov/ radhomel papers/ bo 82.39 ) b. pdl, octoher 1994.

[16] Rohert Reed, Paul Marshall, Jim Foney, and Curtis Dunsmore, "Heavy Ion Latch-up Test Results for the Dallas Semiconductor DSI80.3 Addressable Dual Digital Potentiometer," http:/radhome. gsti. nasa. gov/ radhome/ papers/ b043099b.pdi, Junc 1999

[17] R.A. Reed, P.W. Marshall, C.J. Marshall, H.S. Kim, Loc Xuan Nguyen, K.A. Laß3el, "Energy Dependence of Proton Damage in AlGaAs Light-Emitting Diodes," submitted and accepted for oral presentation at the IFEE TNS, December 2000. 